Res. Lett. Inf. Math. Sci., 2003, Vol.5, pp 23-59

Available online at http://iims.massey.ac.nz/research/letters/

\title{
Cubic elliptic functions
}

\author{
Shaun Cooper \\ Institute of Information $\&$ Mathematical Sciences \\ Massey University at Albany, Auckland, New Zealand \\ s.cooper@massey.ac.nz
}

The function:

$$
\Phi(\theta ; q)=\theta+3 \sum_{k=1}^{\infty} \frac{\sin (2 k \theta) q^{k}}{k\left(1+q^{k}+q^{2 k}\right)}
$$

occurs in one of Ramanujan's inversion formulas for elliptic integrals. In this article, a common generalization of the cubic elliptic functions

$$
\begin{aligned}
g_{1}(\theta ; q) & =\frac{1}{6}+\sum_{k=1}^{\infty} \frac{q^{k}}{1+q^{k}+q^{2 k}} \cos k \theta, \\
g_{2}(\theta ; q) & =\frac{1}{2} \frac{\sin \frac{\theta}{2}}{\sin \frac{3 \theta}{2}}+\sum_{k=1}^{\infty} \frac{\chi_{3}(k) q^{k}}{1-q^{k}} \cos k \theta,
\end{aligned}
$$

is given. The function $g_{1}$ is the derivative of Ramanujan's function $\Phi$ (after rescaling), and $\chi_{3}(n)=0,1$ or -1 according as $n \equiv 0,1$ or $2(\bmod 3)$, respectively, and $|q|<1$. Many properties of the common generalization, as well as the functions $g_{1}$ and $g_{2}$, are proved.

\section{Introduction}

Suppose $\operatorname{Re} t>0$ and let $q=e^{-2 \pi t}$. The function

$$
\phi(\theta ; q)=\frac{1}{4} \cot \frac{\theta}{2}+\sum_{n=1}^{\infty} \frac{q^{n}}{1-q^{n}} \sin n \theta
$$

plays an important role in Ramanujan's paper (16). For example, Ramanujan (16, eq. (17)) proved that

$$
\phi(\theta ; q)^{2}=\left(\frac{1}{4} \cot \frac{\theta}{2}\right)^{2}+\sum_{n=1}^{\infty} \frac{q^{n}}{\left(1-q^{n}\right)^{2}} \cos n \theta+\frac{1}{2} \sum_{n=1}^{\infty} \frac{n q^{n}}{1-q^{n}}(1-\cos n \theta),
$$

and he used this to prove many identities for elliptic functions.

Venkatachaliengar (19, p. 42) generalized Ramanujan's formula (1.2). Let

$$
F(x, y ; q)=\prod_{n=1}^{\infty} \frac{\left(1-x y q^{n-1}\right)\left(1-x^{-1} y^{-1} q^{n}\right)\left(1-q^{n}\right)^{2}}{\left(1-x q^{n-1}\right)\left(1-x^{-1} q^{n}\right)\left(1-y q^{n-1}\right)\left(1-y^{-1} q^{n}\right)}
$$


and

$$
\begin{aligned}
\rho(z ; q) & =\frac{1}{2}+\sum_{\substack{n=-\infty \\
n \neq 0}}^{\infty} \frac{z^{n}}{1-q^{n}}, \quad \text { for }|q|<|z|<1, \\
& =\frac{1+z}{2(1-z)}+\sum_{n=1}^{\infty} \frac{q^{n}}{1-q^{n}}\left(z^{n}-z^{-n}\right), \text { for }|q|<|z|<|q|^{-1} .
\end{aligned}
$$

The function $\rho$ is related to the function $\phi$ in (1.1) by

$$
\rho\left(e^{i \theta}\right)=2 i \phi(\theta) .
$$

Venkatachaliengar's generalization of Ramanujan's identity (1.2) is

$$
F(x, y ; q) F(x, z ; q)=x \frac{\partial}{\partial x} F(x, y z ; q)+F(x, y z ; q)(\rho(y ; q)+\rho(z ; q)) .
$$

See $(9$, p. 66), (10, Thm. 2.2), or (19, p. 37, eqs. (3.2), (3.3)). Letting $y \rightarrow 1 / z$ and then setting $x=e^{i \theta}, z=e^{i \alpha}$, we get

$$
F\left(e^{i \theta}, e^{i \alpha} ; q\right) F\left(e^{i \theta}, e^{-i \alpha} ; q\right)=2\left(\phi^{\prime}(\theta ; q)-\phi^{\prime}(\alpha ; q)\right) .
$$

See $(9$, p. 90, eq. (3.23)), (10, (2.41)), (19, p. 112, eq. (6.50)) for the details. This is equivalent to $(20$, p. 451, Ex. 1$)$ :

$$
\wp(\theta)-\wp(\alpha)=-\frac{\sigma(\theta+\alpha) \sigma(\theta-\alpha)}{\sigma^{2}(\theta) \sigma^{2}(\alpha)},
$$

where $\wp$ is the Weierstrass elliptic function with periods $2 \pi$ and $2 \pi i t$, and $\sigma$ is the corresponding Weierstrass sigma function. Ramanujan's formula (1.2) may be obtained by expanding (1.6) in powers of $\alpha$ and extracting coefficients of $\alpha^{0}$. See (19, pp. 42-45, eq. (3.40)).

The aim of this article is to give analagous results for the functions

$$
\begin{aligned}
& g_{1}(\theta ; q)=\frac{1}{6}+\sum_{n=1}^{\infty} \frac{q^{n}}{1+q^{n}+q^{2 n}} \cos n \theta \\
& g_{2}(\theta ; q)=\frac{1}{2} \frac{\sin \frac{\theta}{2}}{\sin \frac{3 \theta}{2}}+\sum_{n=1}^{\infty} \frac{\chi_{3}(n) q^{n}}{1-q^{n}} \cos n \theta
\end{aligned}
$$

The antiderivative of the function $g_{1}$ occurs in one of Ramanujan's inversion formulas for elliptic integrals. Several properties of $g_{1}$ were established by Berndt, Bhargava and Garvan (4). They used the notation $v(z, q)$, where

$$
g_{1}(\theta ; q)=\frac{1}{6} v\left(e^{i \theta}, q\right) .
$$

We shall begin by observing that $g_{1}$ and $g_{2}$ have a common generalization. A number of basic properties of the generalization, and the functions $g_{1}$ and $g_{2}$ are given in Section 2. Power series expansions for $g_{1}$ and $g_{2}$ in terms of the corresponding Eisenstein series are given in Section 3. In Section 4 , we prove the transformation formulas

$$
\begin{aligned}
& g_{1}\left(\theta ; e^{-2 \pi t}\right)=\frac{1}{t \sqrt{3}} g_{2}\left(\frac{i \theta}{3 t} ; e^{-\frac{2 \pi}{3 t}}\right) \\
& g_{2}\left(\theta ; e^{-2 \pi t}\right)=\frac{1}{t \sqrt{3}} g_{1}\left(\frac{i \theta}{t} ; e^{-\frac{2 \pi}{3 t}}\right) .
\end{aligned}
$$

Expanding in powers of $\theta$, we obtain transformation formulas for various Eisenstein series. A detailed analysis of these Eisenstein series is given in Section 5. 
Connections between the functions $g_{1}, g_{2}$ and the Hirschhorn-Garvan-J. Borwein cubic theta functions are given in Section 6 and connections with the Weierstrass $\wp$ function are given in Section 7. We indicate how to obtain addition formulas for $g_{1}$ and $g_{2}$ in Section 7. Various formulas for $g_{1}, g_{2} g_{1}^{\prime}$ and $g_{2}^{\prime}$, involving infinite products, are given in Section 8 .

An analogue of Venkatachaliengar's formula (1.5) for the function $G$ is given in Section 9, and an analogue of (1.6) is given in Section 10. Fourier series for $g_{1}^{2}$ and $g_{2}^{2}$ are given as a consequence. These are analogues of Ramanujan's formula (1.2).

In Section 11, we introduce the cubic transcendentals $Z$ and $X$. We express Ramanujan's Eisenstein series $P(q), Q(q), R(q)$, as well as $P\left(q^{3}\right), Q\left(q^{3}\right)$ and $R\left(q^{3}\right)$, in terms of $Z$ and $X$. In Section 12 , the differential equations satisfied by $g_{1}$ and $g_{2}$ are given. Lastly, in Section 13 we prove some recurrence relations for cubic Eisenstein series.

\section{Definitions and basic properties}

\subsection{The functions $F$ and $G$}

Let

$$
F(x, y ; q)=\frac{\left(x y, q x^{-1} y^{-1}, q, q ; q\right)_{\infty}}{\left(x, q x^{-1}, y, q y^{-1} ; q\right)_{\infty}}
$$

Here we are using the standard notation

$$
\begin{gathered}
(x ; q)_{\infty}=\prod_{n=1}^{\infty}\left(1-x q^{n-1}\right), \\
\left(x_{1}, x_{2}, \cdots, x_{n} ; q\right)_{\infty}=\left(x_{1} ; q\right)_{\infty}\left(x_{2} ; q\right)_{\infty} \cdots\left(x_{n} ; q\right)_{\infty} .
\end{gathered}
$$

It is straightforward to check that

$$
\begin{aligned}
& F(x, y ; q)=F(y, x ; q) \\
& F(x, y ; q)=-F\left(x^{-1}, y^{-1} ; q\right) \\
& F(x, y ; q)=x F(x, q y ; q)=y F(q x, y ; q)
\end{aligned}
$$

By Ramanujan's ${ }_{1} \psi_{1}$ summation formula (1, p. 502), (2, Ch. 16, Entry 17), (17, Ch. 16, Entry 17),

$$
F(x, y ; q)=\sum_{n=-\infty}^{\infty} \frac{x^{n}}{1-y q^{n}}, \text { for }|q|<|x|<1
$$

Since $F$ is symmetric in $x$ and $y$,

$$
F(x, y ; q)=\sum_{n=-\infty}^{\infty} \frac{y^{n}}{1-x q^{n}}, \text { for }|q|<|y|<1
$$

Let

$$
G(x, y ; q)=\frac{1}{i \sqrt{3}}\left(F(x, \omega y ; q)-F\left(x, \omega^{2} y ; q\right)\right)
$$

Observe that $G$ is not symmetric in $x$ and $y$. 
Expanding in powers of $x$ using (2.5) gives

$$
\begin{aligned}
G(x, y ; q) & =\frac{1}{i \sqrt{3}} \sum_{n=-\infty}^{\infty}\left(\frac{x^{n}}{1-\omega y q^{n}}-\frac{x^{n}}{1-\omega^{2} y q^{n}}\right) \\
& =\sum_{n=-\infty}^{\infty} \frac{x^{n} q^{n} y}{1+y q^{n}+y^{2} q^{2 n}} \\
& =\frac{y}{1+y+y^{2}}+\sum_{n=1}^{\infty} \frac{x^{n} q^{n} y}{1+y q^{n}+y^{2} q^{2 n}}+\sum_{n=1}^{\infty} \frac{x^{-n} q^{n} y^{-1}}{1+y^{-1} q^{n}+y^{-2} q^{2 n}}
\end{aligned}
$$

This series converges for $|q|<|x|<|q|^{-1}$.

Expanding in powers of $y$ using (2.6) gives

$$
\begin{aligned}
G(x, y ; q) & =\frac{1}{i \sqrt{3}} \sum_{n=-\infty}^{\infty}\left(\frac{\omega^{n} y^{n}}{1-x q^{n}}-\frac{\omega^{2 n} y^{n}}{1-x q^{n}}\right) \\
& =\sum_{n=-\infty}^{\infty} \frac{\chi_{3}(n) y^{n}}{1-x q^{n}} .
\end{aligned}
$$

This converges for $|q|<|y|<1$. Another form may be obtained from this as follows:

$$
\begin{aligned}
G(x, y ; q) & =\sum_{n=1}^{\infty} \frac{\chi_{3}(n) y^{n}}{1-x q^{n}}+\sum_{n=1}^{\infty} \frac{\chi_{3}(-n) y^{-n}}{1-x q^{-n}} \\
& =\sum_{n=1}^{\infty} \frac{\chi_{3}(n) y^{n}\left(1-x q^{n}+x q^{n}\right)}{1-x q^{n}}+\sum_{n=1}^{\infty} \frac{\chi_{3}(n) x^{-1} y^{-n} q^{n}}{1-x^{-1} q^{n}} \\
& =\sum_{n=1}^{\infty} \chi_{3}(n) y^{n}+\sum_{n=1}^{\infty} \chi_{3}(n)\left(\frac{x y^{n} q^{n}}{1-x q^{n}}+\frac{x^{-1} y^{-n} q^{n}}{1-x^{-1} q^{n}}\right) \\
& =\frac{y}{1+y+y^{2}}+\sum_{n=1}^{\infty} \chi_{3}(n)\left(\frac{x y^{n} q^{n}}{1-x q^{n}}+\frac{x^{-1} y^{-n} q^{n}}{1-x^{-1} q^{n}}\right) .
\end{aligned}
$$

This converges for $|q|<|y|<|q|^{-1}$.

Also from (2.9) we have

$$
\begin{aligned}
G(x, y ; q) & =\sum_{n=-\infty}^{\infty} \frac{y^{3 n+1}}{1-x q^{3 n+1}}-\sum_{n=-\infty}^{\infty} \frac{y^{3 n-1}}{1-x q^{3 n-1}} \\
& =y F\left(q x, y^{3} ; q^{3}\right)-y^{-1} F\left(q^{-1} x, y^{3} ; q^{3}\right) .
\end{aligned}
$$

\subsection{The functions $g_{1}$ and $g_{2}$}

Let

$$
\begin{aligned}
g_{1}(\theta ; q) & =\frac{1}{2} G\left(e^{i \theta}, 1 ; q\right), \\
g_{2}(\theta ; q) & =\frac{1}{2} G\left(1, e^{i \theta} ; q\right) .
\end{aligned}
$$


Equations (2.8) and (2.10) immediately give

$$
\begin{aligned}
g_{1}(\theta) & =\frac{1}{6}+\sum_{n=1}^{\infty} \frac{q^{n}}{1+q^{n}+q^{2 n}} \cos n \theta \\
& =\frac{1}{6}+\frac{1}{2} \sum_{n=1}^{\infty} \chi_{3}(n)\left(\frac{q^{n} e^{i \theta}}{1-q^{n} e^{i \theta}}+\frac{q^{n} e^{-i \theta}}{1-q^{n} e^{-i \theta}}\right), \\
g_{2}(\theta) & =\frac{1}{2} \frac{\sin \frac{\theta}{2}}{\sin \frac{3 \theta}{2}}+\sum_{n=1}^{\infty} \frac{\chi_{3}(n) q^{n}}{1-q^{n}} \cos n \theta \\
& =\frac{1}{2} \frac{\sin \frac{\theta}{2}}{\sin \frac{3 \theta}{2}}+\sum_{n=1}^{\infty}\left(\frac{e^{i \theta} q^{n}}{1+e^{i \theta} q^{n}+e^{2 i \theta} q^{2 n}}+\frac{e^{-i \theta} q^{n}}{1+e^{-i \theta} q^{n}+e^{-2 i \theta} q^{2 n}}\right) .
\end{aligned}
$$

Equations (2.14) and (2.16) are Fourier series for $g_{1}$ and $g_{2}$. They converge for $|q|<\left|e^{i \theta}\right|<|q|^{-1}$, or equivalently, $-2 \pi \operatorname{Re} t<\operatorname{Im} \theta<2 \pi \operatorname{Re} t$. Equations (2.15) and (2.17) are the analytic continuations of $g_{1}$ and $g_{2}$, and are valid for all complex values of $\theta$.

Equation (2.11) gives

$$
\begin{aligned}
& g_{1}(\theta ; q)=\frac{1}{2}\left(F\left(q e^{i \theta}, 1 ; q^{3}\right)-F\left(q^{-1} e^{i \theta}, 1 ; q^{3}\right)\right) \\
& g_{2}(\theta ; q)=\frac{1}{2}\left(e^{i \theta} F\left(q, e^{3 i \theta} ; q^{3}\right)-e^{-i \theta} F\left(q^{-1}, e^{3 i \theta} ; q^{3}\right)\right) .
\end{aligned}
$$

These expressions are valid for all complex values of $\theta$.

The locations of the poles and periodicity properties are readily determined from (2.15) and (2.17).

Theorem 2.20. Let $q=e^{-2 \pi t}$, where $\operatorname{Re} t>0$. Then

1. $g_{1}(\theta+2 \pi ; q)=g_{1}(\theta ; q)$ $g_{1}(\theta+6 \pi i t ; q)=g_{1}(\theta ; q)$.

2. $g_{1}(\theta ; q)$ is meromorphic on $C$, with simple poles at $\theta=2 \pi m+2 \pi i n t, m, n \in Z, n \not \equiv 0$ $(\bmod 3)$, and no other singularities. The residue at each pole is $\frac{1}{2 i} \chi_{3}(n)$.

3. $g_{2}(\theta+2 \pi ; q)=g_{2}(\theta ; q)$ $g_{2}(\theta+2 \pi i t ; q)=g_{2}(\theta ; q)$.

4. $g_{2}(\theta ; q)$ is meromorphic on $C$, with simple poles at $\theta=2 \pi n / 3+2 \pi i m t, m, n \in Z, n \not \equiv 0$ $(\bmod 3)$, and no other singularities. The residue at each pole is $\frac{-1}{2 \sqrt{3}} \chi_{3}(n)$.

\section{Proof}

Let $z=e^{i \theta}$. The Fourier series (2.14) shows $g_{1}(\theta+2 \pi ; q)=g_{1}(\theta ; q)$.

Next, from (2.15) we have

$$
\begin{aligned}
g_{1}( & +6 \pi i t ; q)-g_{1}(\theta ; q) \\
= & \frac{1}{2} \sum_{n=1}^{\infty}\left(\frac{q^{3 n+1} z}{1-q^{3 n+1} z}-\frac{q^{3 n+2} z}{1-q^{3 n+2} z}+\frac{q^{3 n-5} z^{-1}}{1-q^{3 n-5} z^{-1}}-\frac{q^{3 n-4} z^{-1}}{1-q^{3 n-4} z^{-1}}\right) \\
& -\frac{1}{2} \sum_{n=1}^{\infty}\left(\frac{q^{3 n-2} z}{1-q^{3 n-2} z}-\frac{q^{3 n-1} z}{1-q^{3 n-1} z}+\frac{q^{3 n-2} z^{-1}}{1-q^{3 n-2} z^{-1}}-\frac{q^{3 n-1} z^{-1}}{1-q^{3 n-1} z^{-1}}\right) \\
= & \frac{1}{2}\left(\frac{-q z}{1-q z}+\frac{q^{2} z}{1-q^{2} z}+\frac{q^{-2} z^{-1}}{1-q^{-2} z^{-1}}-\frac{q^{-1} z^{-1}}{1-q^{-1} z^{-1}}\right) \\
= & 0 .
\end{aligned}
$$


Equation (2.15) implies that $g_{1}(\theta, q)$ is meromorphic and has simple poles at $\theta=2 \pi m+2 \pi i n t$, $n \not \equiv 0(\bmod 3)$, and no other singularities. The residue at $\theta=2 \pi i t$ may be calculated as follows.

$$
\begin{aligned}
\operatorname{Res}\left(g_{1}(\theta ; q), \theta=2 \pi i t\right) & =\lim _{\theta \rightarrow 2 \pi i t}(\theta-2 \pi i t) g_{1}(\theta ; q) \\
& =\frac{1}{2} \lim _{\theta \rightarrow 2 \pi i t}(\theta-2 \pi i t) \frac{q e^{-i \theta}}{1-q e^{-i \theta}} \\
& =\frac{1}{2} \lim _{\theta \rightarrow 2 \pi i t} \frac{q e^{-i \theta}}{\frac{d}{d \theta}\left(1-q e^{-i \theta}\right)} \\
& =\frac{1}{2 i} .
\end{aligned}
$$

Similarly, $\operatorname{Res}\left(g_{1}(\theta ; q), \theta=4 \pi i t\right)=-\frac{1}{2 i}$. The residue at the singularity $\theta=2 \pi m+2 \pi i n t$ is therefore $\frac{1}{2 i} \chi_{3}(n)$ by the periodicity properties.

The corresponding properties for the function $g_{2}$ may be proved in the same way, using (2.17).

\subsection{The functions $\phi, h_{1}$ and $h_{2}$}

The function $\phi$ is defined by (1.1). Its analytic continuation, quasi-periodicity properties and the location of its singularities are given by

Theorem 2.21. Let $q=e^{-2 \pi t}$, where $\operatorname{Re} t>0$. Then

1.

$$
\phi(\theta ; q)=\frac{1}{4} \cot \frac{\theta}{2}+\frac{1}{2 i} \sum_{n=1}^{\infty}\left(\frac{q^{n} e^{i \theta}}{1-q^{n} e^{i \theta}}-\frac{q^{n} e^{-i \theta}}{1-q^{n} e^{-i \theta}}\right),
$$

valid for all complex values of $\theta$.

2.

$$
\begin{aligned}
\phi(\theta+2 \pi ; q) & =\phi(\theta ; q) \\
\phi(\theta+2 \pi i t ; q) & =\phi(\theta ; q)-\frac{i}{2} .
\end{aligned}
$$

3. The function $\phi(\theta ; q)$ is meromorphic on $C$, with simple poles at $\theta=2 \pi n+2 \pi i m t$, and no other singularities. The residue at each pole is $\frac{1}{2}$.

\section{Proof}

Starting with (1.1) and writing $z=e^{i \theta}$, we have

$$
\begin{aligned}
\phi(\theta ; q) & =\frac{1}{4} \cot \frac{\theta}{2}+\sum_{n=1}^{\infty} \frac{q^{n}}{1-q^{n}} \sin n \theta \\
& =\frac{1}{4} \cot \frac{\theta}{2}+\frac{1}{2 i} \sum_{n=1}^{\infty} \frac{q^{n}}{1-q^{n}}\left(z^{n}-z^{-n}\right) \\
& =\frac{1}{4} \cot \frac{\theta}{2}+\frac{1}{2 i} \sum_{n=1}^{\infty} \sum_{m=1}^{\infty}\left(q^{m n} z^{n}-q^{m n} z^{-n}\right) \\
& =\frac{1}{4} \cot \frac{\theta}{2}+\frac{1}{2 i} \sum_{m=1}^{\infty}\left(\frac{q^{m} z}{1-q^{m} z}-\frac{q^{m} z^{-1}}{1-q^{m} z^{-1}}\right) \\
& =\frac{1}{4} \cot \frac{\theta}{2}+\frac{1}{2 i} \sum_{m=1}^{\infty}\left(\frac{q^{m} e^{i \theta}}{1-q^{m} e^{i \theta}}-\frac{q^{m} e^{-i \theta}}{1-q^{m} e^{-i \theta}}\right)
\end{aligned}
$$


This proves the first part of the theorem.

The quasi-periodicity properties, location of the singularities and values of the residues can be determined from (2.22) using the same procedure as in the proof of Theorem 2.20.

For future reference, we define the functions $h_{1}$ and $h_{2}$ by

$$
\begin{aligned}
h_{1}(\theta ; q) & =\phi^{\prime}(\theta ; q)-\phi^{\prime}\left(\theta ; q^{3}\right) \\
& =\sum_{n=1}^{\infty} \frac{n q^{n}}{1-q^{n}} \cos n \theta-\sum_{n=1}^{\infty} \frac{n q^{3 n}}{1-q^{3 n}} \cos n \theta \\
h_{2}(\theta ; q) & =\phi^{\prime}(\theta ; q)-9 \phi^{\prime}\left(3 \theta ; q^{3}\right) \\
& =\frac{9}{8} \csc ^{2} \frac{3 \theta}{2}-\frac{1}{8} \csc ^{2} \frac{\theta}{2}+\sum_{n=1}^{\infty} \frac{n q^{n}}{1-q^{n}} \cos n \theta-9 \sum_{n=1}^{\infty} \frac{n q^{3 n}}{1-q^{3 n}} \cos 3 n \theta .
\end{aligned}
$$

The analytic continuations, periodicity properties and location of poles follow right away from the definition of these functions and Theorem 2.21.

\subsection{The cubic theta functions}

The cubic theta functions are defined by

$$
\begin{aligned}
a^{\prime}(q, z) & =\sum_{m=-\infty}^{\infty} \sum_{n=-\infty}^{\infty} q^{m^{2}+m n+n^{2}} z^{n}, \\
a(q, z) & =\sum_{m=-\infty}^{\infty} \sum_{n=-\infty}^{\infty} q^{m^{2}+m n+n^{2}} z^{m-n}, \\
b(q, z) & =\sum_{m=-\infty}^{\infty} \sum_{n=-\infty}^{\infty} q^{m^{2}+m n+n^{2}} \omega^{m-n} z^{n}, \\
c(q, z) & =\sum_{m=-\infty}^{\infty} \sum_{n=-\infty}^{\infty} q^{\left(m+\frac{1}{3}\right)^{2}+\left(m+\frac{1}{3}\right)\left(n+\frac{1}{3}\right)+\left(n+\frac{1}{3}\right)^{2}} z^{m-n},
\end{aligned}
$$

where $\omega=\exp (2 \pi i / 3)$ and $|q|<1$. When $z=1$, we will denote the functions $a^{\prime}(q, 1)=a(q, 1)$ simply by $a(q)$. Similarly, we will abbreviate $b(q, 1)$ and $c(q, 1)$ to $b(q)$ and $c(q)$, respectively. The functions $a^{\prime}(q, z), a(q, z), b(q, z)$ and $c(q, z)$ were introduced ${ }^{1}$ by Hirschhorn et. al. (12). They showed $(12,(1.22),(1.23))$ that

$$
\begin{aligned}
& b(q, z)=(q ; q)_{\infty}\left(q^{3} ; q^{3}\right)_{\infty} \frac{(q z ; q)_{\infty}\left(q z^{-1} ; q\right)_{\infty}}{\left(q^{3} z ; q^{3}\right)_{\infty}\left(q^{3} z^{-1} ; q^{3}\right)_{\infty}} \\
& c(q, z)=q^{\frac{1}{3}}(q ; q)_{\infty}\left(q^{3} ; q^{3}\right)_{\infty}\left(1+z+z^{-1}\right) \frac{\left(q^{3} z^{3} ; q^{3}\right)_{\infty}\left(q^{3} z^{-3} ; q^{3}\right)_{\infty}}{(q z ; q)_{\infty}\left(q z^{-1} ; q\right)_{\infty}}
\end{aligned}
$$

\footnotetext{
${ }^{1}$ The function $c(q, z)$ in (12) differs from the one defined here by a factor of $q^{\frac{1}{3}}$.
} 
We also record, for future reference, the properties

$$
\begin{aligned}
a(q) & =\sum_{m=-\infty}^{\infty} \sum_{n=-\infty}^{\infty} q^{m^{2}+m n+n^{2}} \\
& =1+6 \sum_{n=1}^{\infty}\left(\frac{q^{3 n-2}}{1-q^{3 n-2}}-\frac{q^{3 n-1}}{1-q^{3 n-1}}\right) \\
& =1+6 \sum_{n=1}^{\infty} \frac{q^{n}}{1+q^{n}+q^{2 n}}, \\
b(q) & =\sum_{m=-\infty}^{\infty} \sum_{n=-\infty}^{\infty} q^{m^{2}+m n+n^{2}} \omega^{m-n} \\
& =\frac{(q ; q)_{\infty}^{3}}{\left(q^{3} ; q^{3}\right)_{\infty}} \\
c(q) & =\sum_{m=-\infty}^{\infty} \sum_{n=-\infty}^{\infty} q^{\left(m+\frac{1}{3}\right)^{2}+\left(m+\frac{1}{3}\right)\left(n+\frac{1}{3}\right)+\left(n+\frac{1}{3}\right)^{2}} \\
& =3 q^{\frac{1}{3}} \frac{\left(q^{3} ; q^{3}\right)_{\infty}^{3}}{(q ; q)_{\infty}}, \\
a(q)^{3} & =b(q)^{3}+c(q)^{3} .
\end{aligned}
$$

Equations (2.32), (2.35) and (2.37) follow from (2.26) - (2.29), by definition. Equations (2.33) and (2.34) are proved in $(6,(2.21),(2.25))$, and (2.36) and (2.38) follow from (2.30) and (2.31). Proofs of equation (2.39) are given in (5), (6) and (15).

\section{Laurent series expansions}

Theorem 3.1. Define the cubic Bernoulli numbers $s_{n}$ by

$$
\frac{1}{2} \frac{\sinh \frac{\theta}{2}}{\sinh \frac{3 \theta}{2}}=\sum_{n=0}^{\infty} \frac{s_{n}}{n !} \theta^{n} .
$$

Let $q=e^{-2 \pi t}$, where $\operatorname{Re} t>0$. For $n=1,2,3, \cdots$, let

$$
\begin{aligned}
E_{2 n}(q) & =-\frac{B_{2 n}}{4 n}+\sum_{k=1}^{\infty} \frac{k^{2 n-1} q^{k}}{1-q^{k}}, n=1,2,3, \cdots, \\
S_{0}(q) & =\frac{1}{6}+\sum_{k=1}^{\infty} \frac{q^{k}}{1+q^{k}+q^{2 k}}, \\
S_{2 n}(q) & =\sum_{k=1}^{\infty} \frac{k^{2 n} q^{k}}{1+q^{k}+q^{2 k}}, n=1,2,3, \cdots \\
E_{2 n}\left(\chi_{3} ; q\right) & =s_{2 n}+\sum_{k=1}^{\infty} \frac{k^{2 n} \chi_{3}(k) q^{k}}{1-q^{k}}, n=0,1,2, \cdots \\
E_{2 n}^{(1)}(q) & =E_{2 n}(q)-E_{2 n}\left(q^{3}\right), n=1,2,3, \cdots \\
E_{2 n}^{(2)}(q) & =E_{2 n}(q)-3^{2 n} E_{2 n}\left(q^{3}\right), n=1,2,3, \cdots
\end{aligned}
$$

Then

$$
S_{0}(q)=E_{0}\left(\chi_{3} ; q\right)=\frac{a(q)}{6},
$$


and

$$
\begin{aligned}
\phi\left(\theta ; e^{-2 \pi t}\right) & =\frac{1}{2 \theta}+\sum_{n=1}^{\infty} \frac{(-1)^{n-1}}{(2 n-1) !} E_{2 n}(q) \theta^{2 n-1}, \\
g_{1}(\theta ; q) & =\sum_{n=0}^{\infty} \frac{(-1)^{n}}{(2 n) !} S_{2 n}(q) \theta^{2 n} \\
g_{2}(\theta ; q) & =\sum_{n=0}^{\infty} \frac{(-1)^{n}}{(2 n) !} E_{2 n}\left(\chi_{3} ; q\right) \theta^{2 n} \\
h_{1}\left(\theta ; e^{-2 \pi t}\right) & =\sum_{n=0}^{\infty} \frac{(-1)^{n}}{(2 n) !} E_{2 n+2}^{(1)}(q) \theta^{2 n} \\
h_{2}\left(\theta ; e^{-2 \pi t}\right) & =\sum_{n=0}^{\infty} \frac{(-1)^{n}}{(2 n) !} E_{2 n+2}^{(2)}(q) \theta^{2 n} .
\end{aligned}
$$

The series expansion for $\phi$ is valid for $0<|\theta|<\min \{2 \pi,|2 \pi i t+2 \pi k|, k \in Z\}$, and the others for $|\theta|<\min \{2 \pi,|2 \pi i t+2 \pi k|, k \in Z\}$.

\section{Proof}

The first result is a restatement of (2.33) and (2.34). The expansion for $\phi$ follows by expanding (1.1) in powers of $\theta$, with the help of the expansion

$$
\frac{1}{2} \cot \frac{\theta}{2}=\frac{1}{\theta}+\sum_{n=1}^{\infty} \frac{B_{2 n}(-1)^{n}}{(2 n) !} \theta^{2 n-1} .
$$

The expansions for $g_{1}$ and $g_{2}$ follow by expanding the Fourier series (2.14) and (2.16) in powers of $\theta$. The expansions for $h_{1}$ and $h_{2}$ follow from the expansion for $\phi$ by using the definitions (2.24) and $(2.25)$.

Remark 3.8. We shall call the numbers $s_{0}, s_{1}, s_{2}, \cdots$, the cubic Bernoulli numbers. These numbers were introduced by Liu (15, eqs. (1.11), (1.13)), who expressed them in terms of derivatives of the cotangent function evaluated at $\pi / 3$. From the definition, it is clear that $s_{2 n+1}=0$. The first few values of $s_{2 n}$ are:

$$
\begin{array}{lll}
s_{0}=\frac{1}{6}, & s_{2}=-\frac{1}{9}, & s_{4}=\frac{1}{3} \\
s_{6}=-\frac{7}{3}, & s_{8}=\frac{809}{27}, & s_{10}=-\frac{1847}{3}, \\
s_{12}=\frac{55601}{3}, & s_{14}=-\frac{6921461}{3}, & s_{16}=\frac{126235201}{3} .
\end{array}
$$

In Section 5, we will show that $(-1)^{n} s_{2 n}>0$. Observe also that

$$
\frac{1}{2} \frac{\sin \frac{\theta}{2}}{\sin \frac{3 \theta}{2}}=\sum_{n=0}^{\infty} \frac{(-1)^{n} s_{2 n}}{(2 n) !} \theta^{2 n} .
$$

For future reference, we make the definitions

$$
\begin{aligned}
& P(q)=-24 E_{2}(q)=1-24 \sum_{n=1}^{\infty} \frac{n q^{n}}{1-q^{n}} \\
& Q(q)=240 E_{2}(q)=1+240 \sum_{n=1}^{\infty} \frac{n^{3} q^{n}}{1-q^{n}} \\
& R(q)=-504 E_{2}(q)=1-504 \sum_{n=1}^{\infty} \frac{n^{5} q^{n}}{1-q^{n}}
\end{aligned}
$$




\section{The modular transformation}

\subsection{The modular transformation for cubic theta functions}

Theorem 4.1. Suppose $\operatorname{Re} t>0$. Then

$$
\begin{aligned}
a^{\prime}\left(e^{-2 \pi t}, e^{i \theta}\right) & =\frac{1}{t \sqrt{3}} \exp \left(\frac{-\theta^{2}}{6 \pi t}\right) a\left(e^{-\frac{2 \pi}{3 t}}, e^{\frac{\theta}{3 t}}\right) \\
a\left(e^{-2 \pi t}, e^{i \theta}\right) & =\frac{1}{t \sqrt{3}} \exp \left(\frac{-\theta^{2}}{2 \pi t}\right) a^{\prime}\left(e^{-\frac{2 \pi}{3 t}}, e^{\frac{\theta}{t}}\right) \\
b\left(e^{-2 \pi t}, e^{i \theta}\right) & =\frac{1}{t \sqrt{3}} \exp \left(\frac{-\theta^{2}}{6 \pi t}\right) c\left(e^{-\frac{2 \pi}{3 t}}, e^{\frac{\theta}{3 t}}\right) \\
c\left(e^{-2 \pi t}, e^{i \theta}\right) & =\frac{1}{t \sqrt{3}} \exp \left(\frac{-\theta^{2}}{2 \pi t}\right) b\left(e^{-\frac{2 \pi}{3 t}}, e^{\frac{\theta}{t}}\right)
\end{aligned}
$$

\section{Proof}

See (11, Theorem 5.12).

Corollary 4.2. Suppose $\operatorname{Re} t>0$. Then

$$
\begin{aligned}
a\left(e^{-2 \pi t}\right) & =\frac{1}{t \sqrt{3}} a\left(e^{-\frac{2 \pi}{3 t}}\right), \\
b\left(e^{-2 \pi t}\right) & =\frac{1}{t \sqrt{3}} c\left(e^{-\frac{2 \pi}{3 t}}\right) \\
c\left(e^{-2 \pi t}\right) & =\frac{1}{t \sqrt{3}} b\left(e^{-\frac{2 \pi}{3 t}}\right) .
\end{aligned}
$$

Proof

Let $\theta=0$ in Theorem 4.1. Also see $(5,(2.2))$ or (11, Corollary 5.19).

\subsection{The modular transformation for $g_{1}$ and $g_{2}$}

\section{Theorem 4.3.}

$$
\begin{aligned}
& g_{1}\left(\theta ; e^{-2 \pi t}\right)=\frac{1}{t \sqrt{3}} g_{2}\left(\frac{i \theta}{3 t} ; e^{-\frac{2 \pi}{3 t}}\right) \\
& g_{2}\left(\theta ; e^{-2 \pi t}\right)=\frac{1}{t \sqrt{3}} g_{1}\left(\frac{i \theta}{t} ; e^{-\frac{2 \pi}{3 t}}\right) .
\end{aligned}
$$

\section{Proof}

Observe that by Theorem 2.20, the functions $g_{1}\left(\theta ; e^{-2 \pi t}\right)$ and $\frac{1}{t \sqrt{3}} g_{2}\left(\frac{i \theta}{3 t} ; e^{-\frac{2 \pi}{3 t}}\right)$ both have simple poles at $\theta=2 \pi m+2 \pi i n t, m, n \in Z, n \not \equiv 0(\bmod 3)$, and no other singularities. Furthermore, the residue of each function at each pole is $\frac{1}{2 i} \chi_{3}(n)$. Therefore the difference

$$
g_{1}\left(\theta ; e^{-2 \pi t}\right)-\frac{1}{t \sqrt{3}} g_{2}\left(\frac{i \theta}{3 t} ; e^{-\frac{2 \pi}{3 t}}\right)
$$

is entire. Again by Theorem 2.20, the difference is doubly periodic, and therefore by Liouville's theorem, it is a constant. The value of the constant can be found by plugging in a value for $\theta$, for 
example $\theta=0$ :

$$
\begin{aligned}
g_{1}\left(\theta ; e^{-2 \pi t}\right)-\frac{1}{t \sqrt{3}} g_{2}\left(\frac{i \theta}{3 t} ; e^{-\frac{2 \pi}{3 t}}\right) & =g_{1}\left(0 ; e^{-2 \pi t}\right)-\frac{1}{t \sqrt{3}} g_{2}\left(0 ; e^{-\frac{2 \pi}{3 t}}\right) \\
& =\frac{1}{6}\left(a\left(e^{-2 \pi t}\right)-\frac{1}{t \sqrt{3}} a\left(e^{-\frac{2 \pi}{3 t}}\right)\right) \\
& =0,
\end{aligned}
$$

by Lemma (4.2). This proves the first part of the theorem.

The second part follows from the first part by replacing $t$ with $1 / 3 t$, then replacing $\theta$ with $i \theta / t$, rearranging and using the fact that $g_{1}$ and $g_{2}$ are even functions of $\theta$.

\subsection{The modular transformation for $\phi, h_{1}$ and $h_{2}$}

The transformation properties for the functions $h_{1}$ and $h_{2}$ are obtained from the corresponding transformation formula for $\phi$.

Lemma 4.5. Let $\operatorname{Re} t>0$. Then

$$
\phi\left(\theta ; e^{-2 \pi t}\right)=\frac{i}{t} \phi\left(\frac{i \theta}{t} ; e^{-\frac{2 \pi}{t}}\right)-\frac{\theta}{4 \pi t} .
$$

Proof

See $(11,(4.7))$ or $(19$, pp. 32-35).

Theorem 4.6. Suppose $\operatorname{Re} t>0$. Then

$$
\begin{aligned}
& h_{1}\left(\theta ; e^{-2 \pi t}\right)=\frac{1}{9 t^{2}} h_{2}\left(\frac{i \theta}{3 t} ; e^{-\frac{2 \pi}{3 t}}\right)-\frac{1}{6 \pi t}, \\
& h_{2}\left(\theta ; e^{-2 \pi t}\right)=\frac{1}{t^{2}} h_{1}\left(\frac{i \theta}{t} ; e^{-\frac{2 \pi}{3 t}}\right)+\frac{1}{2 \pi t} .
\end{aligned}
$$

\section{Proof}

Differentiating the result in Lemma 4.5 with respect to $\theta$ gives

$$
\phi^{\prime}\left(\theta ; e^{-2 \pi t}\right)=-\frac{1}{t^{2}} \phi^{\prime}\left(\frac{i \theta}{t} ; e^{-\frac{2 \pi}{t}}\right)-\frac{1}{4 \pi t} .
$$

Replacing $t$ with $3 t$ in Lemma 4.5 and then differentiating with respect to $\theta$ gives

$$
\phi^{\prime}\left(\theta ; e^{-6 \pi t}\right)=-\frac{1}{9 t^{2}} \phi^{\prime}\left(\frac{i \theta}{3 t} ; e^{-\frac{2 \pi}{3 t}}\right)-\frac{1}{12 \pi t} .
$$

Therefore, on writing $q=e^{-2 \pi t}$ and $p=e^{-2 \pi / 3 t}$, we have

$$
\begin{aligned}
h_{1}(\theta ; q) & =\phi^{\prime}(\theta ; q)-\phi^{\prime}\left(\theta ; q^{3}\right) \\
& =-\frac{1}{t^{2}} \phi^{\prime}\left(\frac{i \theta}{t} ; p^{3}\right)-\frac{1}{4 \pi t}+\frac{1}{9 t^{2}} \phi^{\prime}\left(\frac{i \theta}{3 t} ; p\right)+\frac{1}{12 \pi t} \\
& =\frac{1}{9 t^{2}}\left(\phi^{\prime}\left(\frac{i \theta}{3 t} ; p\right)-9 \phi^{\prime}\left(\frac{i \theta}{t} ; p^{3}\right)\right)-\frac{1}{6 \pi t} \\
& =\frac{1}{9 t^{2}} h_{2}\left(\frac{i \theta}{3 t} ; p\right)-\frac{1}{6 \pi t} .
\end{aligned}
$$

This proves the first part of the theorem. The second part follows from the first by replacing $t$ with $1 / 3 t$, and then replacing $\theta$ with $\theta /$ it. 


\subsection{Transformation of Eisenstein series}

Corollary 4.7. Suppose $\operatorname{Re} t>0$ and let $q=e^{-2 \pi t}, p=e^{-\frac{2 \pi}{3 t}}$. Then, for $m \geq 0$,

$$
\begin{aligned}
& S_{2 m}(q)=\frac{(-1)^{m} \sqrt{3}}{(3 t)^{2 m+1}} E_{2 m}\left(\chi_{3} ; p\right), \\
& E_{2 m}\left(\chi_{3} ; q\right)=\frac{(-1)^{m}}{t^{2 m+1} \sqrt{3}} S_{2 m}(p) .
\end{aligned}
$$

\section{Proof}

These follow by expanding the equations in Theorem 4.3 in powers of $\theta$ using Theorem 3.1, and equating coefficients of $\theta^{2 m}$.

Remark 4.8. A different proof of Corollary 4.7 was given by Chan and Liu (7). Another proof of this result (for $m \geq 1$ ) will be given in the next section.

Corollary 4.9. Suppose $\operatorname{Re} t>0$ and let $q=e^{-2 \pi t}, p=e^{-2 \pi / 3 t}$. Then

$$
\begin{aligned}
E_{2}(q) & =-\frac{1}{t^{2}} E_{2}\left(p^{3}\right)-\frac{1}{4 t}, \\
E_{2}^{(1)}(q) & =\frac{1}{9 t^{2}} E_{2}^{(2)}(p)-\frac{1}{6 \pi t}, \\
E_{2}^{(2)}(q) & =\frac{1}{t^{2}} E_{2}^{(1)}(p)+\frac{1}{2 \pi t},
\end{aligned}
$$

and for $n=2,3,4, \cdots$, we have

$$
\begin{aligned}
& E_{2 m}(q)=\frac{(-1)^{m}}{t^{2 m}} E_{2 m}\left(p^{3}\right), \\
& E_{2 m}^{(1)}(q)=\frac{(-1)^{(m-1)}}{3^{2 m} t^{2 m}} E_{2 m}^{(2)}(p), \\
& E_{2 m}^{(2)}(q)=\frac{(-1)^{(m-1)}}{t^{2 m}} E_{2 m}^{(1)}(p) .
\end{aligned}
$$

Proof

Substitute the series expansions from Lemma 3.1 into Lemma 4.5 and Theorem 4.6.

\section{More on Eisenstein series}

The Bernoulli numbers $\left\{B_{n}\right\}$ are defined by

$$
\frac{x}{e^{x}-1}=\sum_{n=0}^{\infty} B_{n} \frac{x^{n}}{n !}
$$

and it is well known (for example, see $(1$, p. 12)), that for each positive integer $n$,

$$
\sum_{k=1}^{\infty} \frac{1}{k^{2 n}}=\frac{(-1)^{n+1} 2^{2 n-1}}{(2 n) !} B_{2 n} \pi^{2 n}
$$

The analogous result for the cubic Bernoulli numbers is 
Theorem 5.1. Let the cubic Bernoulli numbers $\left\{s_{n}\right\}$ be defined by

$$
\frac{1}{2} \frac{\sinh \frac{x}{2}}{\sinh \frac{3 x}{2}}=\sum_{n=0}^{\infty} s_{n} \frac{x^{n}}{n !}
$$

or equivalently by

$$
\frac{1}{2} \frac{\sin \frac{x}{2}}{\sin \frac{3 x}{2}}=\sum_{n=0}^{\infty}(-1)^{n} s_{n} \frac{x^{n}}{n !}
$$

Then

1. For all complex numbers $\tau$,

$$
\sum_{k=-\infty}^{\infty} \frac{\chi_{3}(k)}{\tau+k}=\frac{2 \pi}{\sqrt{3}} \frac{\sin \frac{\pi \tau}{3}}{\sin \pi \tau} .
$$

The series on the left converges for all complex values of $\tau$ (except for integer values of the form $3 k \pm 1$, where there are simple poles), but not absolutely. An absolutely convergent series is given by

$$
\sum_{k=-\infty}^{\infty} \chi_{3}(k)\left(\frac{1}{\tau+k}-\frac{1}{k}\right)=\frac{2 \pi}{\sqrt{3}} \frac{\sin \frac{\pi \tau}{3}}{\sin \pi \tau}
$$

2. For each non-negative integer $n$,

$$
\sum_{k=1}^{\infty} \frac{\chi_{3}(k)}{k^{2 n+1}}=\frac{(-1)^{n} 2^{2 n+1}}{3^{2 n+\frac{1}{2}}(2 n) !} s_{2 n} \pi^{2 n+1} .
$$

3. $(-1)^{n} s_{2 n}>0$.

\section{Proof}

Starting with the partial fractions expansion of the cotangent (1, p. 11)

$$
\pi \cot \pi x=\frac{1}{x}+\sum_{n=1}^{\infty}\left(\frac{1}{x+n}+\frac{1}{x-n}\right)
$$

we obtain

$$
\begin{aligned}
\frac{\pi}{3} & \left(\cot \frac{\pi}{3}(\tau+1)-\cot \frac{\pi}{3}(\tau-1)\right) \\
= & \frac{1}{\tau+1}+\left(\frac{1}{\tau+4}+\frac{1}{\tau-2}\right)+\left(\frac{1}{\tau+7}+\frac{1}{\tau-5}\right)+\cdots \\
& -\frac{1}{\tau-1}-\left(\frac{1}{\tau+2}+\frac{1}{\tau-4}\right)-\left(\frac{1}{\tau+5}+\frac{1}{\tau-7}\right)+\cdots \\
= & \sum_{k=-\infty}^{\infty} \frac{\chi_{3}(k)}{\tau+k}
\end{aligned}
$$

Next, using

$$
\cot (x+y)-\cot (x-y)=\frac{2 \sin 2 y}{\cos 2 x-\cos 2 y}
$$

and

$$
\frac{1}{1+2 \cos 2 x}=\frac{\sin x}{\sin 3 x}
$$


we get

$$
\frac{\pi}{3}\left(\cot \frac{\pi}{3}(\tau+1)-\cot \frac{\pi}{3}(\tau-1)\right)=\frac{2 \pi / \sqrt{3}}{1+2 \cos \frac{2 \pi \tau}{3}}=\frac{2 \pi}{\sqrt{3}} \frac{\sin \frac{\pi \tau}{3}}{\sin \pi \tau}
$$

Equating (5.4) and (5.5) proves (5.2).

The series on the left hand side of (5.2) can be seen to be convergent by considering the real and imaginary parts of the terms. It is clear that the series is not absolutely convergent, since $\left|\frac{\chi_{3}(k)}{\tau+k}\right|=O\left(\frac{1}{k}\right)$. The series (5.2) and (5.3) converge to the same value because $\frac{\chi_{3}(k)}{k}=-\frac{\chi_{3}(-k)}{-k}$, and the series in (5.3) converges absolutely because $\left|\chi_{3}(k)\left(\frac{1}{\tau+k}-\frac{1}{k}\right)\right|=O\left(\frac{1}{k^{2}}\right)$. This completes the proof of the first part of the Theorem.

Expanding the left hand side of (5.2) in powers of $\tau$ gives

$$
\begin{aligned}
\sum_{k=-\infty}^{\infty} \frac{\chi_{3}(k)}{\tau+k} & =2 \sum_{k=1}^{\infty} \frac{k \chi_{3}(k)}{k^{2}-\tau^{2}} \\
& =2 \sum_{k=1}^{\infty} \frac{\chi_{3}(k)}{k} \frac{1}{\left(1-\frac{\tau^{2}}{k^{2}}\right)} \\
& =2 \sum_{k=1}^{\infty} \frac{\chi_{3}(k)}{k} \sum_{n=0}^{\infty} \frac{\tau^{2 n}}{k^{2 n}} \\
& =2 \sum_{n=0}^{\infty}\left(\sum_{k=1}^{\infty} \frac{\chi_{3}(k)}{k^{2 n+1}}\right) \tau^{2 n}
\end{aligned}
$$

valid for $|\tau|<1$. Expanding the right hand side of (5.2) in powers of $\tau$ gives

$$
\frac{2 \pi}{\sqrt{3}} \frac{\sin \frac{\pi \tau}{3}}{\sin \pi \tau}=\frac{4 \pi}{\sqrt{3}} \sum_{n=0}^{\infty} \frac{(-1)^{n} s_{2 n}}{(2 n) !}\left(\frac{2 \pi \tau}{3}\right)^{2 n} .
$$

Equating coefficients of $\tau^{2 n}$ in (5.6) and (5.7) completes the proof of the second part of the Theorem. The third part of the Theorem follows immediately from the second part, since the sum of the series is positive.

Lemma 5.8. Suppose $\operatorname{Im} \tau>0$. Then

$$
\begin{aligned}
& \sum_{k=-\infty}^{\infty} \frac{1}{(\tau+k)^{n+1}}=\frac{(-2 \pi i)^{n+1}}{n !} \sum_{k=1}^{\infty} k^{n} e^{2 \pi i \tau k}, \quad \text { if } n \geq 1, \\
& \sum_{k=-\infty}^{\infty} \frac{\chi_{3}(k)}{(\tau+k)^{n+1}}=\frac{2 \pi}{n ! \sqrt{3}}\left(-\frac{2 \pi i}{3}\right)^{n} \sum_{k=1}^{\infty} \chi_{3}(k) k^{n} e^{2 \pi i \tau k / 3}, \quad \text { if } n \geq 0 .
\end{aligned}
$$

\section{Proof}

The first of these is a standard result, for example, see (13, p. 226, eq. (8.9)) or $(14$, p. 65, Th. 
4). We shall prove the second part. By Theorem 5.1 we have, on writing $u=e^{2 \pi i \tau / 3}$,

$$
\begin{aligned}
\sum_{k=-\infty}^{\infty} \frac{\chi_{3}(k)}{\tau+k} & =\frac{2 \pi}{\sqrt{3}} \frac{\sin \frac{\pi \tau}{3}}{\sin \pi \tau} \\
& =\frac{2 \pi}{\sqrt{3}} \frac{\left(u^{1 / 2}-u^{-1 / 2}\right)}{\left(u^{3 / 2}-u^{-3 / 2}\right)} \\
& =\frac{2 \pi}{\sqrt{3}} \frac{u(1-u)}{1-u^{3}} \\
& =\frac{2 \pi}{\sqrt{3}} \sum_{k=1}^{\infty} \chi_{3}(k) u^{k} \\
& =\frac{2 \pi}{\sqrt{3}} \sum_{k=1}^{\infty} \chi_{3}(k) e^{2 \pi i \tau k / 3}
\end{aligned}
$$

This proves the $k=0$ case of the Lemma. The general case follows from this by first rewriting the left hand side as an absolutely convergent series using (5.3):

$$
\sum_{k=-\infty}^{\infty} \chi_{3}(k)\left(\frac{1}{\tau+k}-\frac{1}{k}\right)=\frac{2 \pi}{\sqrt{3}} \sum_{k=1}^{\infty} \chi_{3}(k) e^{2 \pi i \tau / 3}
$$

and then differentiating $n$ times with respect to $\tau$.

Theorem 5.11. Suppose $\operatorname{Re} t>0$, let $\tau=i t, q=e^{-2 \pi t}=e^{2 \pi i \tau}$. Then

$$
\begin{aligned}
\sum_{(m, n) \neq(0,0)} \frac{1}{(m+n \tau)^{2 j}} & =2 \frac{(2 \pi i)^{2 j}}{(2 j-1) !} E_{2 j}(q) \\
\sum_{m=-\infty}^{\infty} \sum_{n \neq 0} \frac{1}{(m+n \tau)^{2 j}} & =2 \frac{(2 \pi i)^{2 j}}{(2 j-1) !} E_{2 j}^{(1)}(q) \\
\sum_{m \neq 0(\bmod 3)} \sum_{n=-\infty}^{\infty} \frac{1}{(m+n \tau)^{2 j}} & =\frac{-2}{(2 j-1) !}\left(\frac{2 \pi i}{3}\right)^{2 j} E_{2 j}^{(2)}\left(q^{\frac{1}{3}}\right) \\
\sum_{m=-\infty}^{\infty} \sum_{n=-\infty}^{\infty} \frac{\chi_{3}(n)}{(m+n \tau)^{2 j+1}} & =-2 \frac{(2 \pi i)^{2 j+1}}{(2 j) !} S_{2 j}(q) \\
\sum_{m=-\infty}^{\infty} \sum_{n=-\infty}^{\infty} \frac{\chi_{3}(m)}{(m+n \tau)^{2 j+1}} & =\frac{-2 i \sqrt{3}}{(2 j) !}\left(\frac{2 \pi i}{3}\right)^{2 j+1} E_{2 j}\left(\chi_{3} ; q^{\frac{1}{3}}\right) .
\end{aligned}
$$

Equations (5.12)-(5.14) hold for $j \geq 2$, and (5.15)-(5.16) hold for $j \geq 1$.

\section{Proof}

The first of these is a standard result, for example, see (13, p. 226, eq. (8.10)). The second and 
third results follow from the first. For example

$$
\begin{aligned}
& \sum_{m=-\infty}^{\infty} \sum_{n \neq 0(\bmod 3)} \frac{1}{(m+n \tau)^{2 j}} \\
& =\sum_{(m, n) \neq(0,0)} \frac{1}{(m+n \tau)^{2 j}}-\sum_{(m, n) \neq(0,0)} \frac{1}{(m+3 n \tau)^{2 j}} \\
& =2 \frac{(2 \pi i)^{2 j}}{(2 j-1) !} E_{2 j}(q)-2 \frac{(2 \pi i)^{2 j}}{(2 j-1) !} E_{2 j}\left(q^{3}\right) \\
& =2 \frac{(2 \pi i)^{2 j}}{(2 j-1) !} E_{2 j}^{(1)}(q),
\end{aligned}
$$

and this is (5.13). Equation (5.14) is obtained similarly.

Next, by Lemma 5.8,

$$
\begin{aligned}
\sum_{m=-\infty}^{\infty} \sum_{n=-\infty}^{\infty} \frac{\chi_{3}(n)}{(m+n \tau)^{2 j+1}} & =2 \sum_{n=1}^{\infty} \chi_{3}(n) \sum_{m=-\infty}^{\infty} \frac{1}{(m+n \tau)^{2 j+1}} \\
& =2 \sum_{n=1}^{\infty} \chi_{3}(n) \sum_{k=1}^{\infty} \frac{(-2 \pi i)^{2 j+1}}{(2 j) !} k^{2 j} q^{n k} \\
& =-2 \frac{(2 \pi i)^{2 j+1}}{(2 j) !} \sum_{k=1}^{\infty} k^{2 j} \sum_{n=1}^{\infty} \chi_{3}(n) q^{n k} \\
& =-2 \frac{(2 \pi i)^{2 j+1}}{(2 j) !} \sum_{k=1}^{\infty} k^{2 j} \frac{q^{k}-q^{2 k}}{1-q^{3 k}} \\
& =-2 \frac{(2 \pi i)^{2 j+1}}{(2 j) !} S_{2 j}(q) .
\end{aligned}
$$

This proves (5.15). Finally, by Theorem 5.1 and Lemma 5.8,

$$
\begin{aligned}
& \sum_{m=-\infty}^{\infty} \sum_{n=-\infty}^{\infty} \frac{\chi_{3}(m)}{(m+n \tau)^{2 j+1}} \\
& =\sum_{m \neq 0} \frac{\chi_{3}(m)}{m^{2 j+1}}+\sum_{m=-\infty}^{\infty} \sum_{n \neq 0} \frac{\chi_{3}(m)}{(m+n \tau)^{2 j+1}} \\
& =2 \sum_{m=1}^{\infty} \frac{\chi_{3}(m)}{m^{2 j+1}}+2 \sum_{n=1}^{\infty}\left(\sum_{m=-\infty}^{\infty} \frac{\chi_{3}(m)}{(m+n \tau)^{2 j+1}}\right) \\
& =2 \sum_{m=1}^{\infty} \frac{\chi_{3}(m)}{m^{2 j+1}}+\frac{4 \pi}{(2 j) ! \sqrt{3}}\left(-\frac{2 \pi i}{3}\right)^{2 j} \sum_{n=1}^{\infty} \sum_{m=1}^{\infty} \chi_{3}(m) m^{2 j} e^{2 \pi i m n \tau / 3} \\
& =\frac{(-1)^{j} 2^{2 j+2} \pi^{2 j+1}}{3^{2 j+\frac{1}{2}}(2 j) !} s_{2 j}+\frac{(-1)^{j} 2^{2 j+2} \pi^{2 j+1}}{3^{2 j+\frac{1}{2}}(2 j) !} \sum_{m=1}^{\infty} \frac{\chi_{3}(m) m^{2 j} q^{\frac{m}{3}}}{1-q^{\frac{m}{3}}} \\
& =\frac{(-1)^{j} 2^{2 j+2} \pi^{2 j+1}}{3^{2 j+\frac{1}{2}}(2 j) !} E_{2 j}\left(\chi_{3} ; q^{\frac{1}{3}}\right),
\end{aligned}
$$

which proves (5.16).

Remark 5.17. Corollary 4.7 in the case $m \geq 1$, and Corollary 4.9 in the case $m \geq 2$ follow immediately from Theorem 5.11. The cases $m=0$ of Corollary 4.7 and $m=1$ of Corollary 4.9 
do not follow from Theorem 5.11, because the double series on the left hand sides do not converge absolutely.

Corollary 5.18. Let $\Omega_{m n}=2 \pi m+2 \pi i n t$ and $\Omega_{m n}^{*}=\frac{2 \pi m}{3}+2 \pi i n t$. Then

$$
\begin{aligned}
& g_{1}(\theta ; q)=g_{1}(0 ; q)+\frac{1}{2 i} \sum_{m=-\infty}^{\infty} \sum_{n=-\infty}^{\infty} \chi_{3}(n)\left[\frac{1}{\theta-\Omega_{m n}}+\frac{\theta}{\left(\Omega_{m n}\right)^{2}}+\frac{1}{\Omega_{m n}}\right], \\
& g_{2}(\theta ; q)=g_{2}(0 ; q)-\frac{1}{2 \sqrt{3}} \sum_{m=-\infty}^{\infty} \sum_{n=-\infty}^{\infty} \chi_{3}(m)\left[\frac{1}{\theta-\Omega_{m n}^{*}}+\frac{\theta}{\left(\Omega_{m n}^{*}\right)^{2}}+\frac{1}{\Omega_{m n}^{*}}\right] .
\end{aligned}
$$

\section{Proof}

The functions $g_{1}^{\prime \prime}(\theta ; q)$ and $\frac{1}{i} \sum_{m=-\infty}^{\infty} \sum_{n=-\infty}^{\infty} \frac{\chi_{3}(n)}{\left(\theta-\Omega_{m n}\right)^{3}}$ are both doubly periodic with periods $2 \pi$ and $6 \pi i t$. Furthermore, they both have poles at $\theta=\Omega_{m n}, n \not \equiv 0(\bmod 3)$, and no other singularities, and the singular parts of both functions at each pole are identical. Consequently their difference is an entire, doubly periodic function, which is therefore constant, i.e.,

$$
g_{1}^{\prime \prime}(\theta ; q)=\frac{1}{i} \sum_{m=-\infty}^{\infty} \sum_{n=-\infty}^{\infty} \frac{\chi_{3}(n)}{\left(\theta-\Omega_{m n}\right)^{3}}+c .
$$

The value of $c$ may be found by plugging in a value for $\theta$, for example $\theta=0$. Using Theorems 3.1 and 5.11, we obtain $c=0$.

Applying $\int_{0}^{\theta} d \theta$ to both sides and using the fact that $g_{1}^{\prime}(0 ; q)=0$, we get

$$
g_{1}^{\prime}(\theta ; q)=-\frac{1}{2 i} \sum_{m=-\infty}^{\infty} \sum_{n=-\infty}^{\infty} \chi_{3}(n)\left[\frac{1}{\left(\theta-\Omega_{m n}\right)^{2}}-\frac{1}{\left(\Omega_{m n}\right)^{2}}\right] .
$$

Applying $\int_{0}^{\theta} d \theta$ again we complete the proof of the first part of the theorem.

The second part may be proved similarly, or obtained from the first part using the modular transformation.

\section{Connection between cubic elliptic functions and cubic theta functions}

The cubic elliptic functions $g_{1}(\theta ; q), g_{2}(\theta ; q)$ are related to the cubic theta functions $b(q, z), c(q, z)$ as follows.

\section{Theorem 6.1.}

$$
\begin{aligned}
& g_{1}(\theta ; q)=\frac{1}{4} \frac{(q ; q)_{\infty}^{2}\left(q^{3} ; q^{3}\right)_{\infty}^{2}}{\left(q^{2} ; q^{2}\right)_{\infty}\left(q^{6} ; q^{6}\right)_{\infty}} \frac{b\left(q,-e^{i \theta}\right)}{b\left(q, e^{i \theta}\right)}-\frac{1}{12} \frac{b(q)^{2}}{b\left(q^{2}\right)} \\
& g_{2}(\theta ; q)=\frac{1}{2} q^{\frac{1}{2}} \frac{(q ; q)_{\infty}^{2}\left(q^{3} ; q^{3}\right)_{\infty}^{2}}{\left(q^{\frac{1}{2}} ; q^{\frac{1}{2}}\right)_{\infty}\left(q^{\frac{3}{2}} ; q^{\frac{3}{2}}\right)_{\infty}} e^{i \theta} \frac{c\left(q, q^{\frac{1}{2}} e^{i \theta}\right)}{c\left(q, e^{i \theta}\right)}-\frac{1}{6} \frac{c(q)^{2}}{c\left(q^{\frac{1}{2}}\right)}
\end{aligned}
$$

\section{Proof}

As usual, let $q=e^{-2 \pi t}, \operatorname{Re} t>0$. Let us put

$$
B(\theta ; q)=\frac{b\left(q,-e^{i \theta}\right)}{b\left(q, e^{i \theta}\right)} .
$$


Clearly $B(\theta ; q)$ is periodic with period $2 \pi$. Next, from $(2.28)$ or $(2.30)$ we find $(12,(1.17))$ that

$$
b(q, z)=z^{2} q^{3} b\left(q, z q^{3}\right),
$$

and therefore $B(\theta ; q)$ is periodic with period $6 \pi i t$.

From $(2.30)$, we see that $B(\theta ; q)$ has simple poles at $\theta=2 \pi m+2 \pi i n t, m, n \in Z, n \not \equiv 0(\bmod 3)$, and no other singularities. We calculate the residue at $2 \pi i t$ :

$$
\begin{aligned}
& \operatorname{Res}(B(\theta ; q) ; \theta=2 \pi i t) \\
& =\lim _{\theta \rightarrow 2 \pi i t}(\theta-2 \pi i t) \frac{b\left(e^{-2 \pi t},-e^{i \theta}\right)}{b\left(e^{-2 \pi t}, e^{i \theta}\right)} \\
& =\lim _{\theta \rightarrow 2 \pi i t} \frac{(\theta-2 \pi i t)}{\left(1-e^{-2 \pi t-i \theta}\right)} \frac{\left(-q e^{i \theta} ; q\right)_{\infty}\left(-q e^{-i \theta} ; q\right)_{\infty}}{\left(-q^{3} e^{i \theta} ; q^{3}\right)_{\infty}\left(-q^{3} e^{-i \theta} ; q^{3}\right)_{\infty}} \frac{\left(q^{3} e^{i \theta} ; q^{3}\right)_{\infty}\left(q^{3} e^{-i \theta} ; q^{3}\right)_{\infty}}{\left(q e^{i \theta} ; q\right)_{\infty}\left(q^{2} e^{-i \theta} ; q\right)_{\infty}} \\
& =-i \frac{\left(-q^{2} ; q\right)_{\infty}(-1 ; q)_{\infty}}{\left(-q^{4} ; q^{3}\right)_{\infty}\left(-q^{2} ; q^{3}\right)_{\infty}} \frac{\left(q^{4} ; q^{3}\right)_{\infty}\left(q^{2} ; q^{3}\right)_{\infty}}{\left(q^{2} ; q\right)_{\infty}(q ; q)_{\infty}} \\
& =-2 i \frac{\left(q^{2} ; q^{2}\right)_{\infty}\left(q^{6} ; q^{6}\right)_{\infty}}{(q ; q)_{\infty}^{2}\left(q^{3} ; q^{3}\right)_{\infty}^{2}},
\end{aligned}
$$

after simplification. Similarly,

$$
\operatorname{Res}(B(\theta ; q) ; \theta=4 \pi i t)=2 i \frac{\left(q^{2} ; q^{2}\right)_{\infty}\left(q^{6} ; q^{6}\right)_{\infty}}{(q ; q)_{\infty}^{2}\left(q^{3} ; q^{3}\right)_{\infty}^{2}},
$$

and by the periodicity properties of $B$, we obtain

$$
\operatorname{Res}(B(\theta ; q) ; \theta=2 \pi m+2 \pi i n t)=\frac{2}{i} \chi_{3}(n) \frac{\left(q^{2} ; q^{2}\right)_{\infty}\left(q^{6} ; q^{6}\right)_{\infty}}{(q ; q)_{\infty}^{2}\left(q^{3} ; q^{3}\right)_{\infty}^{2}} .
$$

By Theorem 2.20, it follows that

$$
g_{1}(\theta ; q)-\frac{1}{4} \frac{(q ; q)_{\infty}^{2}\left(q^{3} ; q^{3}\right)_{\infty}^{2}}{\left(q^{2} ; q^{2}\right)_{\infty}\left(q^{6} ; q^{6}\right)_{\infty}} \frac{b\left(q,-e^{i \theta}\right)}{b\left(q, e^{i \theta}\right)}
$$

is doubly periodic and entire. Therefore by Liouville's theorem it is constant. Letting $\theta=0$ we find that the value of the constant is given by

$$
\begin{aligned}
g_{1} & (0 ; q)-\frac{1}{4} \frac{(q ; q)_{\infty}^{2}\left(q^{3} ; q^{3}\right)_{\infty}^{2}}{\left(q^{2} ; q^{2}\right)_{\infty}\left(q^{6} ; q^{6}\right)_{\infty}} \frac{b(q,-1)}{b(q, 1)} \\
& =\frac{1}{6} a(q)-\frac{1}{4} \frac{(q ; q)_{\infty}^{2}\left(q^{3} ; q^{3}\right)_{\infty}^{2}}{\left(q^{2} ; q^{2}\right)_{\infty}\left(q^{6} ; q^{6}\right)_{\infty}} \frac{(-q ; q)_{\infty}^{2}}{\left(-q^{3} ; q^{3}\right)_{\infty}^{2}} \frac{\left(q^{3} ; q^{3}\right)_{\infty}^{2}}{(q ; q)_{\infty}^{2}} \\
& =\frac{1}{6} a(q)-\frac{1}{4} \frac{\left(q^{3} ; q^{3}\right)_{\infty}^{6}}{(q ; q)_{\infty}^{2}} \frac{\left(q^{2} ; q^{2}\right)_{\infty}}{\left(q^{6} ; q^{6}\right)_{\infty}^{3}} \\
& =\frac{1}{6} a(q)-\frac{1}{12} \frac{c(q)^{2}}{c\left(q^{2}\right)} \\
& =-\frac{1}{12} \frac{b(q)^{2}}{b\left(q^{2}\right)}
\end{aligned}
$$

The last step follows by $(12,(1.29))$. This completes the proof of the first part of the Theorem. The second part may be proved similarly. Alternatively, it can be deduced from the first part by applying the modular transformation and using Theorems 4.1 and 4.3.

Remark 6.4. Equation (6.2) was proved by Berndt et. al. (4, Lemma 8.2). The proof we have given here is simpler. 


\section{Connection between cubic elliptic functions and the Weierstrass $\wp$ function}

In this section, we establish connections between the cubic elliptic functions $g_{1}, g_{2}$ and the Weierstrass $\wp$ function. The results in this section will be used in Section 12 to obtain differential equations for $g_{1}$ and $g_{2}$, and also in Section 13 to prove recurrence relations for the Eisenstein series $S_{2 n}(q)$ and $E_{2 n}\left(\chi_{3} ; q\right)$.

\section{Theorem 7.1.}

$$
\begin{aligned}
\left(g_{1}(\theta ; q)-g_{1}(0 ; q)\right)\left(\phi^{\prime}\left(\theta ; q^{3}\right)-\phi^{\prime}\left(2 \pi i t ; q^{3}\right)\right) & =\frac{S_{2}(q)}{4} \\
\left(g_{2}(\theta ; q)-g_{2}(0 ; q)\right)\left(\phi^{\prime}(\theta ; q)-\phi^{\prime}\left(\frac{2 \pi}{3} ; q\right)\right) & =\frac{E_{2}\left(\chi_{3} ; q\right)}{4} .
\end{aligned}
$$

\section{Proof}

Let

$$
\begin{aligned}
\alpha(\theta) & =g_{1}(\theta ; q)-g_{1}(0 ; q), \\
\beta(\theta) & =\phi^{\prime}\left(\theta ; q^{3}\right)-\phi^{\prime}\left(2 \pi i t ; q^{3}\right) .
\end{aligned}
$$

By Theorems 2.20 and 3.1,

- $\alpha$ is periodic with periods $2 \pi$ and $6 \pi i t$;

- $\alpha$ has simple poles at $\theta=2 \pi m+2 \pi i n t, n \not \equiv 0(\bmod 3)$, and no other singularities;

- $\alpha$ has zeros of order 2 at $\theta=2 \pi m+6 \pi i n t$, and no other zeros.

Similarly, by Theorem 2.21, and the fact that $\phi^{\prime}$ is an even function, we have that

- $\beta$ is periodic with periods $2 \pi$ and $6 \pi i t$;

- $\beta$ has simple zeros at $\theta=2 \pi m+2 \pi i n t, n \not \equiv 0(\bmod 3)$, and no other zeros;

- $\beta$ has poles of order 2 at $\theta=2 \pi m+6 \pi i n t$, and no other singularities.

It follows that the product $\alpha(\theta) \beta(\theta)$ is a doubly periodic function with no zeros or poles, and therefore is a constant. Letting $\theta \rightarrow 0$ and using the expansions in Theorem 3.1, we find that the value of the constant is $S_{2}(q) / 4$. This proves the first part of the theorem.

The second part may either be proved similarly, or obtained from the first part using the transformation $t \rightarrow 1 / 3 t$.

The Weierstrass $\wp$ function with periods $\omega_{1}$ and $\omega_{2}$ is defined by

$$
\wp\left(\theta ; \omega_{1}, \omega_{2}\right)=\frac{1}{\theta^{2}}+\sum_{(m, n) \neq(0,0)}\left(\frac{1}{\left(\theta-m \omega_{1}-n \omega_{2}\right)^{2}}-\frac{1}{\left(m \omega_{1}+n \omega_{2}\right)^{2}}\right) .
$$

It can be shown that (9), (10)

$$
\wp(\theta ; 2 \pi, 2 \pi i t)=-2 \phi^{\prime}(\theta ; q)-\frac{P(q)}{12},
$$

where $q=e^{-2 \pi t}$. Using this in Theorem 7.1, we obtain 


\section{Theorem 7.3.}

$$
\begin{aligned}
& g_{1}(\theta ; q)-g_{1}(0 ; q)=\frac{S_{2}(q)}{2(\wp(2 \pi i t ; 2 \pi, 6 \pi i t)-\wp(\theta ; 2 \pi, 6 \pi i t))}, \\
& g_{2}(\theta ; q)-g_{2}(0 ; q)=\frac{E_{2}\left(\chi_{3} ; q\right)}{2\left(\wp\left(\frac{2 \pi}{3} ; 2 \pi, 2 \pi i t\right)-\wp(\theta ; 2 \pi, 2 \pi i t)\right)} .
\end{aligned}
$$

A slightly different formulation of Theorem 7.3 will be given in Section 12 .

We conclude this Section by observing that addition formulas for $g_{1}$ and $g_{2}$ which express $g_{1}(\alpha+\beta)$ (resp. $g_{2}(\alpha+\beta)$ ) in terms of $g_{1}(\alpha), g_{1}(\beta), g_{1}^{\prime}(\alpha)$ and $g_{1}^{\prime}(\beta)$, (resp. $g_{2}(\alpha), g_{2}(\beta), g_{2}^{\prime}(\alpha)$ and $\left.g_{2}^{\prime}(\beta)\right)$ can be obtained from Theorem 7.3 and the addition formula for the Weierstrass $\wp$ function:

$$
\wp(\alpha+\beta)+\wp(\alpha)+\wp(\beta)=\frac{1}{4}\left(\frac{\wp^{\prime}(\alpha)-\wp^{\prime}(\beta)}{\wp(\alpha)-\wp(\beta)}\right)^{2} .
$$

\section{Infinite product representations}

The functions $g_{1}$ and $g_{2}$ may be expressed as differences of infinite products.

\section{Theorem 8.1.}

$$
\begin{aligned}
& g_{1}(\theta ; q)=\frac{(q ; q)_{\infty}^{3}}{\left(q^{3} ; q^{3}\right)_{\infty}}\left(\frac{\sin \left(\frac{\theta}{2}+\frac{\pi}{3}\right)}{\sin \frac{\theta}{2}} \prod_{n=1}^{\infty} \frac{1-2 q^{n} \cos \left(\theta+\frac{2 \pi}{3}\right)+q^{2 n}}{1-2 q^{n} \cos \theta+q^{2 n}}\right.\left.-\frac{\sin \left(\frac{\theta}{2}-\frac{\pi}{3}\right)}{\sin \frac{\theta}{2}} \prod_{n=1}^{\infty} \frac{1-2 q^{n} \cos \left(\theta-\frac{2 \pi}{3}\right)+q^{2 n}}{1-2 q^{n} \cos \theta+q^{2 n}}\right) \\
& g_{2}(\theta ; q)=\frac{3\left(q^{3} ; q^{3}\right)_{\infty}^{3}}{(q ; q)_{\infty}}\left(e^{-i \theta} \frac{\left(q^{2} e^{3 i \theta}, q e^{-3 i \theta} ; q^{3}\right)_{\infty}}{\left(q^{3} e^{3 i \theta}, e^{-3 i \theta} ; q^{3}\right)_{\infty}}\right. \\
&\left.-e^{i \theta} \frac{\left(q e^{3 i \theta}, q^{2} e^{-3 i \theta} ; q^{3}\right)_{\infty}}{\left(e^{3 i \theta}, q^{3} e^{-3 i \theta} ; q^{3}\right)_{\infty}}\right) .
\end{aligned}
$$

\section{Proof}

Equation (8.2) follows from (2.1), (2.7) and (2.12), and some simplification. Equation (8.3) is obtained from (2.1) and (2.19). Alternatively, (8.3) can be proved by applying the modular transformation $t \rightarrow \frac{1}{3 t}$ to $(8.2)$.

The derivatives $g_{1}^{\prime}$ and $g_{2}^{\prime}$ can both be written as single infinite products.

\section{Theorem 8.4.}

$$
\begin{aligned}
\frac{d}{d \theta} g_{1}(\theta ; q)= & -q(q ; q)_{\infty}\left(q^{3} ; q^{3}\right)_{\infty}^{3} \sin \theta \frac{\left(q^{3} e^{2 i \theta}, q^{3} e^{-2 i \theta} ; q^{3}\right)_{\infty}\left(q^{3} e^{i \theta}, q^{3} e^{-i \theta} ; q^{3}\right)_{\infty}^{2}}{\left(q e^{i \theta}, q e^{-i \theta} ; q\right)_{\infty}^{2}} \\
\frac{d}{d \theta} g_{2}(\theta ; q)= & (q ; q)_{\infty}^{3}\left(q^{3} ; q^{3}\right)_{\infty} \sin \theta\left(q e^{2 i \theta}, q e^{-2 i \theta} ; q\right)_{\infty} \\
& \times \frac{\sin ^{2} \frac{\theta}{2}}{\sin ^{2} \frac{3 \theta}{2}} \frac{\left(q e^{i \theta}, q e^{-i \theta} ; q\right)_{\infty}^{2}}{\left(q^{3} e^{3 i \theta}, q^{3} e^{-3 i \theta} ; q^{3}\right)_{\infty}^{2}} .
\end{aligned}
$$




\section{Proof}

$$
\begin{aligned}
\frac{d g_{1}}{d \theta} & =-\sum_{n=1}^{\infty} \frac{n q^{n} \sin n \theta}{1+q^{n}+q^{2 n}} \\
& =-\frac{1}{2 i} \sum_{n=1}^{\infty} \frac{n\left(q^{n}-q^{2 n}\right)\left(e^{i n \theta}-e^{-i n \theta}\right)}{1-q^{3 n}} \\
& =-\frac{1}{2 i} \sum_{n=1}^{\infty}\left(\frac{n q^{n} e^{i n \theta}}{1-q^{3 n}}-\frac{n q^{n} e^{-i n \theta}}{1-q^{3 n}}-\frac{n q^{2 n} e^{i n \theta}}{1-q^{3 n}}+\frac{n q^{2 n} e^{-i n \theta}}{1-q^{3 n}}\right) .
\end{aligned}
$$

Observe that

$$
\begin{aligned}
\sum_{n=1}^{\infty} \frac{n q^{n} e^{i n \theta}}{1-q^{3 n}} & =\sum_{n=1}^{\infty} \frac{n\left(q^{n}-q^{4 n}+q^{4 n}\right) e^{i n \theta}}{1-q^{3 n}} \\
& =\sum_{n=1}^{\infty} n q^{n} e^{i n \theta}+\sum_{n=1}^{\infty} \frac{n q^{4 n} e^{i n \theta}}{1-q^{3 n}} \\
& =\frac{q e^{i \theta}}{\left(1-q e^{i \theta}\right)^{2}}+\sum_{n=1}^{\infty} \frac{n q^{4 n} e^{i n \theta}}{1-q^{3 n}} \\
& =-\frac{1}{4} \csc ^{2} \frac{\theta+2 \pi i t}{2}+\sum_{n=1}^{\infty} \frac{n q^{4 n} e^{i n \theta}}{1-q^{3 n}}
\end{aligned}
$$

and similarly

$$
\sum_{n=1}^{\infty} \frac{n q^{2 n} e^{i n \theta}}{1-q^{3 n}}=-\frac{1}{4} \csc ^{2} \frac{\theta+4 \pi i t}{2}+\sum_{n=1}^{\infty} \frac{n q^{5 n} e^{i n \theta}}{1-q^{3 n}} .
$$

Substituting these into (8.5) and using (1.1) we get

$$
\begin{aligned}
\frac{d g_{1}}{d \theta}= & -\frac{1}{2 i}\left(-\frac{1}{4} \csc ^{2} \frac{\theta+2 \pi i t}{2}+2 \sum_{n=1}^{\infty} \frac{n q^{3 n}}{1-q^{3 n}} \cos n(\theta+2 \pi i t)\right) \\
& +\frac{1}{2 i}\left(-\frac{1}{4} \csc ^{2} \frac{\theta+4 \pi i t}{2}+2 \sum_{n=1}^{\infty} \frac{n q^{3 n}}{1-q^{3 n}} \cos n(\theta+4 \pi i t)\right) \\
= & i\left(\phi^{\prime}\left(\theta+2 \pi i t ; e^{-6 \pi t}\right)-\phi^{\prime}\left(\theta+4 \pi i t ; e^{-6 \pi t}\right)\right) .
\end{aligned}
$$

Finally, using (1.6) and (2.1), this becomes

$$
\begin{aligned}
\frac{d g_{1}}{d \theta}= & \frac{i}{2} F\left(e^{i \theta-4 \pi t}, e^{i \theta-2 \pi t} ; e^{-6 \pi t}\right) F\left(e^{-i \theta+4 \pi t}, e^{i \theta-2 \pi t} ; e^{-6 \pi t}\right) \\
= & \frac{i}{2} \frac{\left(q^{3} e^{2 i \theta}, e^{-2 i \theta}, q^{-1}, q^{4}, q^{3}, q^{3}, q^{3}, q^{3} ; q^{3}\right)_{\infty}}{\left(q^{2} e^{i \theta}, q e^{-i \theta}, q e^{i \theta}, q^{2} e^{-i \theta}, q^{-2} e^{-i \theta}, q^{5} e^{i \theta}, q e^{i \theta}, q^{2} e^{-i \theta} ; q^{3}\right)_{\infty}} \\
= & \frac{i}{2}\left(1-e^{-2 i \theta}\right) \frac{\left(1-q^{-1}\right)}{(1-q)} \frac{\left(1-q^{2} e^{i \theta}\right)}{\left(1-q^{-2} e^{-i \theta}\right)} \\
& \quad \times \frac{\left(q^{3} e^{2 i \theta}, q^{3} e^{-2 i \theta} ; q^{3}\right)_{\infty}(q ; q)_{\infty}\left(q^{3} ; q^{3}\right)_{\infty}^{3}}{\left(q e^{i \theta}, q^{2} e^{i \theta}, q e^{-i \theta}, q^{2} e^{-i \theta} ; q^{3}\right)_{\infty}^{2}} \\
& \\
= & -q(q ; q)_{\infty}\left(q^{3} ; q^{3}\right)_{\infty}^{3} \sin \theta \frac{\left(q^{3} e^{2 i \theta}, q^{3} e^{-2 i \theta} ; q^{3}\right)_{\infty}\left(q^{3} e^{i \theta}, q^{3} e^{-i \theta} ; q^{3}\right)_{\infty}^{2}}{\left(q e^{i \theta}, q e^{-i \theta} ; q\right)_{\infty}^{2}} .
\end{aligned}
$$

This proves the first part of the theorem.

The second part may be proved similarly, or by using the modular transformation $t \rightarrow 1 / 3 t$. 
Remark 8.6. The first part of Theorem 8.4 was proved by Berndt et. al. (4, Lemma 8.5), using the ${ }_{6} \psi_{6}$ summation formula. The proof we have given here is simpler.

\section{$9 \quad$ A multiplicative identity for $G$}

In this section, we give a multiplicative identity for $G$, which is analogous to Venkatachaliengar's identity (1.5) for $F$. We begin with some preparatory lemmas.

Lemma 9.1.

$$
\begin{gathered}
F(x, y ; q)+F(x, \omega y ; q)+F\left(x, \omega^{2} y ; q\right)=3 F\left(x, y^{3} ; q^{3}\right) . \\
\rho(x ; q)+\rho(\omega x ; q)+\rho\left(\omega^{2} x ; q\right)=3 \rho\left(x^{3} ; q^{3}\right),
\end{gathered}
$$

\section{Proof}

The first of these follows immediately from the series representation (2.5). The second follows from (1.3).

Lemma 9.2. Let

$$
f(x)=\sum_{n=-\infty}^{\infty} c_{n} x^{n}
$$

in some annulus $r_{1}<|x|<r_{2}$. Let

$$
\operatorname{sift}(f(x) ; x, m, k)=\sum_{n=-\infty}^{\infty} c_{m n+k} x^{m n+k} .
$$

Then

$$
\begin{aligned}
\operatorname{sift}(\rho(x ; q) ; x, 3,0) & =\rho\left(x^{3} ; q^{3}\right), \\
\operatorname{sift}(\rho(x ; q) ; x, 3,1) & =x F\left(x^{3}, q ; q^{3}\right), \\
\operatorname{sift}(\rho(x ; q) ; x, 3,2) & =x^{2} F\left(x^{3}, q^{2} ; q^{3}\right), \\
\operatorname{sift}(F(x, y ; q) ; y, 3,0) & =F\left(x, y^{3} ; q^{3}\right), \\
\operatorname{sift}(F(x, y ; q) ; y, 3,1) & =y F\left(q x, y^{3} ; q^{3}\right), \\
\operatorname{sift}(F(x, y ; q) ; y, 3,2) & =y^{2} F\left(q^{2} x, y^{3} ; q^{3}\right) .
\end{aligned}
$$

\section{Proof}

These all follow directly from the series representations (2.5) and (1.3).

Lemma 9.3. Suppose the series $\sum_{n=-\infty}^{\infty} a_{n} x^{n}$ and $\sum_{n=-\infty}^{\infty} b_{n} x^{n}$ both converge in the annulus $r_{1}<|x|<r_{2}$. Then in this annulus,

$$
\begin{gathered}
\sum_{n=-\infty}^{\infty} a_{n} x^{n} \sum_{n=-\infty}^{\infty} b_{n} x^{n}+\sum_{n} a_{n} \omega^{2 n} x^{n} \sum_{n} \omega^{n} b_{n} x^{n}+\sum_{n} a_{n} \omega^{n} x^{n} \sum_{n} \omega^{2 n} b_{n} x^{n} \\
=3\left[\sum_{n} a_{3 n} x^{3 n} \sum_{n} b_{3 n} x^{3 n}+\sum_{n} a_{3 n+1} x^{3 n+1} \sum_{n} b_{3 n+1} x^{3 n+1}\right. \\
\left.+\sum_{n} a_{3 n+2} x^{3 n+2} \sum_{n} b_{3 n+2} x^{3 n+2}\right] .
\end{gathered}
$$




\section{Proof}

Write

$$
\sum a_{n} x^{n}=\sum a_{3 n} x^{3 n}+\sum a_{3 n+1} x^{3 n+1}+\sum a_{3 n+2} x^{3 n+2}
$$

and sift each of the other series $\sum a_{n} \omega^{n} x^{n}, \sum a_{n} \omega^{2 n} x^{n}, \sum b_{n} x^{n}, \sum b_{n} \omega^{n} x^{n}$ and $\sum b_{n} \omega^{2 n} x^{n}$ similarly. The result follows by expanding and simplifying.

\section{Theorem 9.4.}

$$
\begin{aligned}
G(x, y ; q) G(x, z ; q) & \\
= & x \frac{\partial}{\partial x}\left(F(x, y z ; q)-F\left(x, y^{3} z^{3} ; q^{3}\right)\right) \\
& +\left(F(x, y z ; q)-F\left(x, y^{3} z^{3} ; q^{3}\right)\right)\left(\rho\left(y^{3} ; q^{3}\right)+\rho\left(z^{3} ; q^{3}\right)\right) \\
& -y z F\left(q x, y^{3} z^{3} ; q^{3}\right)\left(y F\left(q, y^{3} ; q^{3}\right)+z F\left(q, z^{3} ; q^{3}\right)\right) \\
& -y^{2} z^{2} F\left(q^{2} x, y^{3} z^{3} ; q^{3}\right)\left(y^{2} F\left(q^{2}, y^{3} ; q^{3}\right)+z^{2} F\left(q^{2}, z^{3} ; q^{3}\right)\right) .
\end{aligned}
$$

\section{Proof}

Using the definition (2.7) and the multiplicative identity (1.5), we obtain

$$
\begin{aligned}
G(x, y ; q) G(x, z ; q) \\
=-\frac{1}{3}\left(F(x, \omega y ; q)-F\left(x, \omega^{2} y ; q\right)\right)\left(F(x, \omega z ; q)-F\left(x, \omega^{2} z ; q\right)\right) \\
=-\frac{1}{3}\left(F(x, \omega y ; q) F(x, \omega z ; q)+F\left(x, \omega^{2} y ; q\right) F\left(x, \omega^{2} z ; q\right)\right. \\
\left.\quad-F\left(x, \omega^{2} y ; q\right) F(x, \omega z ; q)-F(x, \omega y ; q) F\left(x, \omega^{2} z ; q\right)\right) \\
=-\frac{1}{3} x \frac{\partial}{\partial x}\left(F(x, \omega y z ; q)+F\left(x, \omega^{2} y z ; q\right)-2 F(x, y z ; q)\right) \\
\quad-\frac{1}{3} F\left(x, \omega^{2} y z ; q\right)(\rho(\omega y ; q)+\rho(\omega z ; q)) \\
\quad-\frac{1}{3} F(x, \omega y z ; q)\left(\rho\left(\omega^{2} y ; q\right)+\rho\left(\omega^{2} z ; q\right)\right) \\
\quad-\frac{1}{3} F(x, y z ; q)\left(\rho(\omega y ; q)+\rho\left(\omega^{2} y ; q\right)+\rho(\omega z ; q)+\rho\left(\omega^{2} z ; q\right)\right) .
\end{aligned}
$$

Applying Lemmas 9.1, 9.2 and 9.3 to this gives

$$
\begin{aligned}
G(x, y ; q) & G(x, z ; q) \\
=x & \frac{\partial}{\partial x}\left(F(x, y z ; q)-F\left(x, y^{3} z^{3} ; q^{3}\right)\right) \\
& +\frac{1}{3} F(x, y z ; q)(\rho(y ; q)+\rho(z ; q)) \\
& -F\left(x, y^{3} z^{3} ; q^{3}\right)\left(\rho\left(y^{3} ; q^{3}\right)+\rho\left(z^{3} ; y^{3}\right)\right) \\
& -y z F\left(q x, y^{3} z^{3} ; q^{3}\right)\left(y F\left(q, y^{3} ; q^{3}\right)+z F\left(q, z^{3} ; q^{3}\right)\right) \\
& -y^{2} z^{2} F\left(q^{2} x, y^{3} z^{3} ; q^{3}\right)\left(y^{2} F\left(q^{2}, y^{3} ; q^{3}\right)+z^{2} F\left(q^{2}, z^{3} ; q^{3}\right)\right) \\
& +\frac{1}{3} F(x, y z ; q)\left(3 \rho\left(y^{3} ; q^{3}\right)-\rho(y ; q)+3 \rho\left(z^{3} ; q^{3}\right)-\rho(z ; q)\right) .
\end{aligned}
$$

Simplifying, we complete the proof. 


\section{Squares}

An important special case of Theorem 9.4 is the limiting case $y \rightarrow 1 / z$. We begin by proving a number of preliminary lemmas which will be useful in computing this limit. Let

$$
\begin{aligned}
& \Omega(x ; q)=x \frac{d}{d x} \rho(x ; q) \\
& =\sum_{\substack{n=-\infty \\
n \neq 0}}^{\infty} \frac{n x^{n}}{1-q^{n}}, \quad \text { if }|q|<|x|<1, \\
& =\frac{x}{(1-x)^{2}}+\sum_{n=1}^{\infty} \frac{n q^{n}\left(x^{n}+x^{-n}\right)}{1-q^{n}}, \quad \text { if }|q|<|x|<|q|^{-1}, \\
& \Lambda(x ; q)=x \frac{d}{d x} F\left(q, x ; q^{3}\right) \\
& =\sum_{\substack{n=-\infty \\
n \neq 0}}^{\infty} \frac{n x^{n}}{1-q^{3 n+1}}, \quad \text { if }\left|q^{3}\right|<|x|<1, \\
& =\frac{x}{(1-x)^{2}}+\sum_{n=1}^{\infty}\left(\frac{n q^{3 n+1} x^{n}}{1-q^{3 n+1}}+\frac{n q^{3 n-1} x^{-n}}{1-q^{3 n-1}}\right), \quad \text { if }|q|^{3}<|x|<|q|^{-3} \text {. }
\end{aligned}
$$

Observe that, from (1.1), (2.24) and (2.25), we have

$$
\begin{aligned}
\Omega\left(e^{i \theta} ; q\right) & =2 \phi^{\prime}(\theta ; q), \\
\Omega\left(e^{i \theta} ; q\right)-\Omega\left(e^{i \theta} ; q^{3}\right) & =2 h_{1}(\theta ; q), \\
\Omega\left(e^{i \alpha} ; q\right)-9 \Omega\left(e^{3 i \alpha} ; q^{3}\right) & =2 h_{2}(\alpha ; q) .
\end{aligned}
$$

Lemma 10.6.

$$
\begin{gathered}
\lim _{y \rightarrow 1 / z} x \frac{\partial}{\partial x} F(x, y z ; q)=\Omega(x ; q), \\
\lim _{y \rightarrow 1 / z} x \frac{\partial}{\partial x} F\left(x, y^{3} z^{3} ; q^{3}\right)=\Omega\left(x ; q^{3}\right) .
\end{gathered}
$$

\section{Proof}

The first of these follows by expanding $F$ in powers of $x$ using (2.5), computing the partial derivative and then evaluating the limit. The second part follows from the first part, by replacing $y, z$ and $q$ by their cubes.

\section{Lemma 10.7.}

$$
\begin{aligned}
\lim _{t \rightarrow 1}(1-t) F(x, t ; q) & =1 \\
\lim _{y \rightarrow 1 / z}\left(1-y^{3} z^{3}\right) F(x, y z ; q) & =3 \\
\lim _{y \rightarrow 1 / z}\left(1-y^{3} z^{3}\right) F\left(x, y^{3} z^{3} ; q\right) & =1 .
\end{aligned}
$$

\section{Proof}

The first part follows from the definition (2.1). The second and third parts follow using $1-y^{3} z^{3}=$ $(1-y z)\left(1+y z+y^{2} z^{2}\right)$ and simple changes of variable. 


\section{Lemma 10.8.}

$$
\begin{gathered}
\lim _{y \rightarrow 1 / z} \frac{\rho\left(y^{3} ; q^{3}\right)+\rho\left(z^{3} ; q^{3}\right)}{1-y^{3} z^{3}}=-\Omega\left(z^{3} ; q^{3}\right), \\
\lim _{y \rightarrow 1 / z} \frac{F\left(q, z^{3} ; q^{3}\right)-F\left(q, y^{-3} ; q^{3}\right)}{1-y^{3} z^{3}}=-\Lambda\left(z^{3} ; q\right) .
\end{gathered}
$$

\section{Proof}

From (1.4), we obtain $\rho(t ; q)=-\rho\left(t^{-1} ; q\right)$. Therefore, putting $w=y^{-1}$ we get

$$
\begin{aligned}
\lim _{y \rightarrow 1 / z} \frac{\rho\left(y^{3} ; q^{3}\right)+\rho\left(z^{3} ; q^{3}\right)}{1-y^{3} z^{3}} & =-\lim _{w \rightarrow z} w^{3} \frac{\rho\left(z^{3} ; q^{3}\right)-\rho\left(w^{3} ; q^{3}\right)}{z^{3}-w^{3}} \\
& =-\left.z^{3} \frac{d}{d t} \rho\left(t ; q^{3}\right)\right|_{t=z^{3}}=-\Omega\left(z^{3} ; q^{3}\right) .
\end{aligned}
$$

Similarly,

$$
\begin{aligned}
\lim _{y \rightarrow 1 / z} \frac{F\left(q, z^{3} ; q^{3}\right)-F\left(q, y^{-3} ; q^{3}\right)}{1-y^{3} z^{3}} \\
\quad=\lim _{w \rightarrow z}-w^{3} \frac{F\left(q, z^{3} ; q^{3}\right)-F\left(q, w^{3} ; q^{3}\right)}{z^{3}-w^{3}} \\
\quad=-\left.z^{3} \frac{d}{d t} F\left(q, t ; q^{3}\right)\right|_{t=z^{3}}=-\Lambda\left(z^{3} ; q\right) .
\end{aligned}
$$

\section{Lemma 10.9.}

$$
\begin{aligned}
& \lim _{y \rightarrow 1 / z} y z^{2} F\left(q x, y^{3} z^{3} ; q^{3}\right) F\left(q, z^{3} ; q^{3}\right)+y^{4} z^{2} F\left(q^{2} x, y^{3} z^{3} ; q^{3}\right) F\left(q^{2}, y^{3} ; q^{3}\right) \\
& \quad=-z \Lambda\left(z^{3} ; q\right)+z F\left(q, z^{3} ; q^{3}\right)\left(G(x, 1 ; q)-\frac{1}{3}\right) \\
& \lim _{y \rightarrow 1 / z} y^{2} z F\left(q x, y^{3} z^{3} ; q^{3}\right) F\left(q, y^{3} ; q^{3}\right)+y^{2} z^{4} F\left(q^{2} x, y^{3} z^{3} ; q^{3}\right) F\left(q^{2}, z^{3} ; q^{3}\right) \\
& \quad=-z^{-1} \Lambda\left(z^{-3} ; q\right)+z^{-1} F\left(q, z^{-3} ; q^{3}\right)\left(G(x, 1 ; q)-\frac{1}{3}\right) .
\end{aligned}
$$

\section{Proof}

First, using (2.3) and (2.4), we have

$$
F\left(q^{2}, y^{3} ; q^{3}\right)=-y^{-3} F\left(q, y^{-3} ; q^{3}\right)
$$


Using this, together with Lemma 10.8, we obtain

$$
\begin{aligned}
& \lim _{y \rightarrow 1 / z} y z^{2} F\left(q x, y^{3} z^{3} ; q^{3}\right) F\left(q, z^{3} ; q^{3}\right)+y^{4} z^{2} F\left(q^{2} x, y^{3} z^{3} ; q^{3}\right) F\left(q^{2}, y^{3} ; q^{3}\right) \\
& =\lim _{y \rightarrow 1 / z} y z^{2}\left(F\left(q x, y^{3} z^{3} ; q^{3}\right) F\left(q, z^{3} ; q^{3}\right)-F\left(q^{2} x, y^{3} z^{3} ; q^{3}\right) F\left(q, y^{-3} ; q^{3}\right)\right) \\
& =z \lim _{y \rightarrow 1 / z} F\left(q x, y^{3} z^{3} ; q^{3}\right)\left(1-y^{3} z^{3}\right)\left(\frac{F\left(q, z^{3} ; q^{3}\right)-F\left(q, y^{-3} ; q^{3}\right)}{1-y^{3} z^{3}}\right) \\
& \quad+z \lim _{y \rightarrow 1 / z} F\left(q, y^{-3} ; q^{3}\right)\left(F\left(q x, y^{3} z^{3} ; q^{3}\right)-F\left(q^{2} x, y^{3} z^{3} ; q^{3}\right)\right) \\
& =\quad-z \Lambda\left(z^{3} ; q\right)+z F\left(q, z^{3} ; q^{3}\right) \sum_{n \neq 0}\left(\frac{q^{n} x^{n}}{1-q^{3 n}}-\frac{q^{2 n} x^{n}}{1-q^{3 n}}\right) \\
& =\quad-z \Lambda\left(z^{3} ; q\right)+z F\left(q, z^{3} ; q^{3}\right) \sum_{n \neq 0} \frac{x^{n} q^{n}}{1+q^{n}+q^{2 n}} \\
& =-z \Lambda\left(z^{3} ; q\right)+z F\left(q, z^{3} ; q^{3}\right)\left(G(x, 1 ; q)-\frac{1}{3}\right) .
\end{aligned}
$$

This proves the first part. The second part follows from this by change of variable.

Lemma 10.10.

$$
\begin{gathered}
z F\left(q, z^{3} ; q^{3}\right)+z^{-1} F\left(q, z^{-3} ; q^{3}\right)=G(1, z ; q) \\
z \Lambda\left(z^{3} ; q\right)+z^{-1} \Lambda\left(z^{-3} ; q\right)=\frac{1}{3} \Omega(z ; q)-\Omega\left(z^{3} ; q^{3}\right)-\frac{1}{3} G(1, z ; q) .
\end{gathered}
$$

\section{Proof}

The first of these follows from (2.11), using (2.2). The second is proved by series manipulations. From (10.1) and (10.2), we have

$$
\begin{aligned}
& \frac{1}{3} \Omega(z ; q)-\Omega\left(z^{3} ; q^{3}\right)-z \Lambda\left(z^{3} ; q\right)-z^{-1} \Lambda\left(z^{-3} ; q\right) \\
&=\frac{1}{3} \frac{z}{(1-z)^{2}}+\frac{1}{3} \sum_{n=1}^{\infty} \frac{n q^{n}}{1-q^{n}}\left(z^{n}+z^{-n}\right) \\
& \quad-\frac{z^{3}}{\left(1-z^{3}\right)^{2}}-\sum_{n=1}^{\infty} \frac{n q^{3 n}}{1-q^{3 n}}\left(z^{3 n}+z^{-3 n}\right) \\
& \quad-\frac{z^{4}}{\left(1-z^{3}\right)^{2}}-\sum_{n=1}^{\infty}\left(\frac{n q^{3 n+1} z^{3 n+1}}{1-q^{3 n+1}}+\frac{n q^{3 n-1} z^{-3 n+1}}{1-q^{3 n-1}}\right) \\
& \quad-\frac{z^{-4}}{\left(1-z^{-3}\right)^{2}}-\sum_{n=1}^{\infty}\left(\frac{n q^{3 n+1} z^{-3 n-1}}{1-q^{3 n+1}}+\frac{n q^{3 n-1} z^{3 n-1}}{1-q^{3 n-1}}\right) \\
&=\frac{1}{3} \frac{z}{(1-z)^{2}}-\frac{z^{3}}{\left(1-z^{3}\right)^{2}}-\frac{z^{4}}{\left(1-z^{3}\right)^{2}}-\frac{z^{-4}}{\left(1-z^{-3}\right)^{2}} \\
&+ \frac{1}{3} \sum_{n=1}^{\infty} \frac{(3 n-2) q^{3 n-2}}{1-q^{3 n-2}}\left(z^{3 n-2}+z^{-(3 n-2)}\right) \\
&+\frac{1}{3} \sum_{n=1}^{\infty} \frac{(3 n-1) q^{3 n-1}}{1-q^{3 n-1}}\left(z^{3 n-1}+z^{-(3 n-1)}\right) \\
&-\sum_{n=0}^{\infty} \frac{n q^{3 n+1}}{1-q^{3 n+1}}\left(z^{3 n+1}+z^{-(3 n+1)}\right)-\sum_{n=1}^{\infty} \frac{n q^{3 n-1}}{1-q^{3 n-1}}\left(z^{3 n-1}+z^{-(3 n-1)}\right) .
\end{aligned}
$$


Simplifying, we obtain

$$
\begin{aligned}
& \frac{1}{3} \Omega(z ; q)-\Omega\left(z^{3} ; q^{3}\right)-z \Lambda\left(z^{3} ; q\right)-z^{-1} \Lambda\left(z^{-3} ; q\right) \\
& =\frac{z}{3\left(1+z+z^{2}\right)}+\frac{1}{3} \sum_{n=1}^{\infty} \frac{q^{3 n-2}}{1-q^{3 n-2}}\left(z^{3 n-2}+z^{-(3 n-2)}\right) \\
& \quad-\frac{1}{3} \sum_{n=1}^{\infty} \frac{q^{3 n-1}}{1-q^{3 n-1}}\left(z^{3 n-1}+z^{-(3 n-1)}\right) \\
& =\frac{z}{3\left(1+z+z^{2}\right)}+\frac{1}{3} \sum_{n=1}^{\infty} \frac{\chi_{3}(n) q^{n}}{1-q^{n}}\left(z^{n}+z^{-n}\right) \\
& =\frac{1}{3} G(1, z ; q),
\end{aligned}
$$

by $(2.10)$.

\section{Theorem 10.11.}

$$
G\left(e^{i \theta}, e^{i \alpha} ; q\right) G\left(e^{i \theta}, e^{-i \alpha} ; q\right)+4 g_{1}(\theta ; q) g_{2}(\alpha ; q)=2 h_{1}(\theta ; q)+\frac{2}{3} h_{2}(\alpha ; q) .
$$

\section{Proof}

First rewrite Theorem 9.4 in the form

$$
\begin{aligned}
G(x, y ; q) G(x, z ; q) \\
=\quad x \frac{\partial}{\partial x}\left(F(x, y z ; q)-F\left(x, y^{3} z^{3} ; q^{3}\right)\right) \\
\quad+\left(F(x, y z ; q)-F\left(x, y^{3} z^{3} ; q^{3}\right)\right)\left(\rho\left(y^{3} ; q^{3}\right)+\rho\left(z^{3} ; q^{3}\right)\right) \\
\quad-\left(y^{2} z F\left(q x, y^{3} z^{3} ; q^{3}\right) F\left(q, y^{3} ; q^{3}\right)+y^{2} z^{4} F\left(q^{2} x, y^{3} z^{3} ; q^{3}\right) F\left(q^{2}, z^{3} ; q^{3}\right)\right) \\
\quad-\left(y z^{2} F\left(q x, y^{3} z^{3} ; q^{3}\right) F\left(q, z^{3} ; q^{3}\right)+y^{4} z^{2} F\left(q^{2} x, y^{3} z^{3} ; q^{3}\right) F\left(q^{2}, y^{3} ; q^{3}\right)\right) .
\end{aligned}
$$

Now take the limit as $y \rightarrow 1 / z$, using Lemmas $10.6-10.9$, to get

$$
\begin{aligned}
& G(x, z ; q) G\left(x, z^{-1} ; q\right) \\
& \quad=\quad \Omega(x ; q)-\Omega\left(x ; q^{3}\right)-2 \Omega\left(z^{3} ; q^{3}\right) \\
& \quad+z \Lambda\left(z^{3} ; q\right)-z F\left(q, z^{3} ; q^{3}\right)\left(G(x, 1 ; q)-\frac{1}{3}\right) \\
& \quad+z^{-1} \Lambda\left(z^{-3} ; q\right)-z^{-1} F\left(q, z^{-3} ; q^{3}\right)\left(G(x, 1 ; q)-\frac{1}{3}\right) .
\end{aligned}
$$

Apply Lemma 10.10 to this, and simplify to get

$$
\begin{aligned}
& G(x, z ; q) G\left(x, z^{-1} ; q\right) \\
& \quad=\quad \Omega(x ; q)-\Omega\left(x ; q^{3}\right)+\frac{1}{3} \Omega(z ; q)-3 \Omega\left(z^{3} ; q^{3}\right)-G(x, 1 ; q) G(1, z ; q) .
\end{aligned}
$$

Setting $x=e^{i \theta}, z=e^{i \alpha}$, and using the definitions (2.12), (2.13), (10.4) and (10.5), we complete the proof.

Theorem 10.11 immediately implies the following results of Liu (15, Theorems 5 and 7): 


\section{Corollary 10.12.}

$$
\begin{aligned}
& \left(g_{1}(\theta ; q)+\frac{a(q)}{12}\right)^{2}=\frac{h_{1}(\theta)}{2}+\frac{9 P\left(q^{3}\right)-P(q)}{144}+\frac{a(q)^{2}}{144}, \\
& \left(g_{2}(\theta ; q)+\frac{a(q)}{12}\right)^{2}=\frac{h_{2}(\theta)}{6}+\frac{P\left(q^{3}\right)-P(q)}{48}+\frac{a(q)^{2}}{144} .
\end{aligned}
$$

\section{Proof}

Take $\alpha=0$ and $\theta=0$ in Theorem 10.11, respectively, use (2.24) and (2.25) and complete the square.

\section{Corollary 10.13.}

$$
\begin{aligned}
a(q)^{2} & =\frac{3}{2} P\left(q^{3}\right)-\frac{1}{2} P(q), \\
E_{4}^{(1)}(q) & =6 S_{0}(q) S_{2}(q), \\
E_{4}^{(2)}(q) & =18 E_{0}\left(\chi_{3} ; q\right) E_{2}\left(\chi_{3} ; q\right) .
\end{aligned}
$$

\section{Proof}

Letting $\theta=0$ in either part of Corollary 10.12, and using (2.24) (or (2.25)), we get

$$
\frac{a(q)^{2}}{16}=\frac{9 P\left(q^{3}\right)-P(q)}{144}+\frac{P\left(q^{3}\right)-P(q)}{48}+\frac{a(q)^{2}}{144} .
$$

Simplifying, we get the first part.

The second and third parts follow by equating coefficients of $\theta^{2}$ in Corollary 10.12.

Remark 10.14. Corollary 10.13 was given by Ramanujan (16, eq. (19)). He obtained it by putting $\theta=2 \pi / 3$ in his identity (1.2). A more general result than Corollary 10.13 can be obtained by equating coefficients of $\theta^{2 n}$. This will be given in Section 13.

\section{Corollary 10.15.}

$$
G\left(e^{i \theta}, e^{i \alpha} ; e^{-2 \pi t}\right) G\left(e^{i \theta}, e^{-i \alpha} ; e^{-2 \pi t}\right)=\frac{1}{3 t^{2}} G\left(e^{\frac{\alpha}{t}}, e^{\frac{\theta}{3 t}} ; e^{-\frac{2 \pi}{3 t}}\right) G\left(e^{\frac{\alpha}{t}}, e^{-\frac{\theta}{3 t}} ; e^{-\frac{2 \pi}{3 t}}\right) .
$$

\section{Proof}

By Theorem 10.11, followed by Theorems 4.3 and 4.6, and then Theorem 10.11 again, we obtain

$$
\begin{aligned}
G\left(e^{i \theta}, e^{i \alpha} ; e^{-2 \pi t}\right) G\left(e^{i \theta}, e^{-i \alpha} ; e^{-2 \pi t}\right) \\
=\quad 2 h_{1}\left(\theta ; e^{-2 \pi t}\right)+\frac{2}{3} h_{2}\left(\alpha ; e^{-2 \pi t}\right)-4 g_{1}\left(\theta ; e^{-2 \pi t}\right) g_{2}\left(\alpha ; e^{-2 \pi t}\right) \\
=2\left(\frac{1}{9 t^{2}} h_{2}\left(\frac{i \theta}{3 t} ; e^{-\frac{2 \pi}{3 t}}\right)-\frac{1}{6 \pi t}\right)+\frac{2}{3}\left(\frac{1}{t^{2}} h_{1}\left(\frac{i \alpha}{t} ; e^{-\frac{2 \pi}{3 t}}\right)+\frac{1}{2 \pi t}\right) \\
\quad \quad-\frac{4}{3 t^{2}} g_{2}\left(\frac{i \theta}{3 t} ; e^{-\frac{2 \pi}{3 t}}\right) g_{1}\left(\frac{i \alpha}{t} ; e^{-\frac{2 \pi}{3 t}}\right) \\
=\frac{1}{3 t^{2}}\left(2 h_{1}\left(\frac{i \alpha}{t} ; e^{-\frac{2 \pi}{3 t}}\right)+\frac{2}{3} h_{2}\left(\frac{i \theta}{3 t} ; e^{-\frac{2 \pi}{3 t}}\right)-4 g_{1}\left(\frac{i \alpha}{t} ; e^{-\frac{2 \pi}{3 t}}\right) g_{2}\left(\frac{i \theta}{3 t} ; e^{-\frac{2 \pi}{3 t}}\right)\right) \\
=\frac{1}{3 t^{2}} G\left(e^{\frac{\alpha}{t}}, e^{\frac{\theta}{3 t}} ; e^{-\frac{2 \pi}{3 t}}\right) G\left(e^{\frac{\alpha}{t}}, e^{-\frac{\theta}{3 t}} ; e^{-\frac{2 \pi}{3 t}}\right) .
\end{aligned}
$$




\section{The transcendentals $Z$ and $X$}

Definition 11.1. Let

$$
\begin{aligned}
Z=Z(q) & =a(q), \\
X=X(q) & =\frac{c(q)^{3}}{a(q)^{3}} .
\end{aligned}
$$

The main result of the section is Theorem 11.11, which expresses various Eisenstein series in terms of $Z$ and $X$. We begin with some lemmas.

\section{Lemma 11.2.}

$$
\begin{aligned}
1-X(q) & =\frac{b(q)^{3}}{a(q)^{3}} \\
Z\left(e^{-2 \pi t}\right) & =\frac{1}{t \sqrt{3}} Z\left(e^{-\frac{2 \pi}{3 t}}\right), \\
X\left(e^{-2 \pi t}\right) & =1-X\left(e^{-\frac{2 \pi}{3 t}}\right) .
\end{aligned}
$$

\section{Proof}

The first part follows from equation (2.39), and the other two parts follow from Lemma 4.2.

\section{Lemma 11.3.}

$$
\begin{aligned}
& \left(\frac{1}{8} \cot ^{2} \frac{\theta}{2}+\frac{1}{12}+\sum_{n=1}^{\infty} \frac{n q^{n}}{1-q^{n}}(1-\cos n \theta)\right)^{2} \\
= & \left(\frac{1}{8} \cot ^{2} \frac{\theta}{2}+\frac{1}{12}\right)^{2}+\frac{1}{12} \sum_{n=1}^{\infty} \frac{n^{3} q^{n}}{1-q^{n}}(5+\cos n \theta) .
\end{aligned}
$$

\section{Proof}

This was given by Ramanujan (18, eq. (18)). It is equivalent to the differential equation satisfied by the Weierstrass $\wp$ function:

$$
\wp^{\prime \prime}(z)=6 \wp^{2}(z)-g_{2} / 2 .
$$

\section{Lemma 11.4.}

$$
\begin{aligned}
& a \frac{\left(a^{2}, q a^{-2} ; q\right)_{\infty}(q ; q)_{\infty}^{6}}{\left(a, q a^{-1} ; q\right)_{\infty}^{4}} \\
& \quad=\frac{a(1+a)}{(1-a)^{3}}+\sum_{m=1}^{\infty} \frac{m^{2} q^{m}}{1-q^{m}}\left(a^{m}-a^{-m}\right)
\end{aligned}
$$

\section{Proof}

Multiply both sides of (1.6) by $e^{i \alpha} /\left(e^{i \theta}-e^{i \alpha}\right)$, take the limit as $\theta \rightarrow \alpha$, and finally put $a=e^{i \alpha}$. This is equivalent to $(20$, p. 459 , ex. 24$)$.

\section{Lemma 11.5.}

$$
\begin{aligned}
b(q)^{3} & =-9 E_{2}\left(\chi_{3} ; q\right) \\
c(q)^{3} & =27 S_{2}(q) \\
a(q)^{4} & =\frac{1}{10}\left(Q(q)+9 Q\left(q^{3}\right)\right) .
\end{aligned}
$$




\section{Proof}

Let $a=e^{2 \pi i / 3}$ in Lemma 11.4 and simplify to obtain (11.6). Replace $q$ with $q^{3}$ in Lemma 11.4, let $a=q$ and simplify to obtain (11.7). Take $\theta=2 \pi / 3$ in Lemma 11.3 and simplify to get

$$
\left(\frac{3}{2} P\left(q^{3}\right)-\frac{1}{2} P(q)\right)^{2}=\frac{1}{10}\left(Q(q)+9 Q\left(q^{3}\right)\right) .
$$

Now apply Corollary 10.13 on the left to complete the proof of (11.8).

Remark 11.9. Equation (11.7) was given by Ramanujan; see (4, Theorem 8.7). Equation (11.8) was given by Berndt et. al. (4, Corollary 4.6). Equations (11.6) and (11.7) were given without proof by J. M. and P. B. Borwein (5, Remark 2.4 (iii)). All of (11.6)-(11.8) were given by Liu (15, eqs. (1.15), (1.16) and (1.19)). The proof of Lemma 11.5 that we have outlined above is substantially the same as Liu's.

\section{Theorem 11.10.}

$$
\begin{aligned}
q(q ; q)_{\infty}^{24} & =\frac{1}{27} Z^{12} X(1-X)^{3}, \\
q^{3}\left(q^{3} ; q^{3}\right)_{\infty}^{24} & =\frac{1}{3^{9}} Z^{12} X^{3}(1-X), \\
q \frac{d X}{d q} & =Z^{2} X(1-X), \\
\frac{3}{2} P\left(q^{3}\right)-\frac{1}{2} P(q) & =Z^{2}, \\
P(q) & =Z^{2}(1-4 X)+12 Z X(1-X) \frac{d Z}{d X}, \\
P\left(q^{3}\right) & =Z^{2}\left(1-\frac{4}{3} X\right)+4 Z X(1-X) \frac{d Z}{d X}, \\
Q(q) & =Z^{4}(1+8 X), \\
Q\left(q^{3}\right) & =Z^{4}\left(1-\frac{8}{9} X\right), \\
R(q) & =Z^{6}\left(1-20 X-8 X^{2}\right), \\
R\left(q^{3}\right) & =Z^{6}\left(1-\frac{4}{3} X+\frac{8}{27} X^{2}\right) .
\end{aligned}
$$

\section{Proof}

Equations (11.11) and (11.12) follow from Definition 11.1, Lemma 11.2 and the infinite product formulas (2.36) and (2.38).

Taking the logarithm of (11.11) and applying $q \frac{d}{d q}$ gives

$$
1-24 \sum_{n=1}^{\infty} \frac{n q^{n}}{1-q^{n}}=q \frac{d}{d q} \log \left(\frac{1}{27} Z^{12} X(1-X)^{3}\right)
$$

which is equivalent to

$$
P(q)=q \frac{d X}{d q}\left(\frac{12}{Z} \frac{d Z}{d X}+\frac{1}{X}-\frac{3}{1-X}\right) .
$$

Applying the same procedure to (11.12) leads to

$$
3 P\left(q^{3}\right)=q \frac{d X}{d q}\left(\frac{12}{Z} \frac{d Z}{d X}+\frac{3}{X}-\frac{1}{1-X}\right) .
$$


Subtracting (11.21) from (11.22) and dividing by 2 gives

$$
\frac{1}{2}\left(3 P\left(q^{3}\right)-P(q)\right)=q \frac{d X}{d q}\left(\frac{1}{X}+\frac{1}{1-X}\right) .
$$

Simplifying using Corollary 10.13 we obtain

$$
q \frac{d X}{d q}=Z^{2} X(1-X)
$$

This proves (11.13).

Equation (11.14) is just a restatement of the first part of Corollary 10.13. Equations (11.15) and (11.16) are obtained by substituting (11.13) into (11.21) and (11.22).

By (3.3), (3.5), Corollary 10.13 and Lemma 11.5, we have

$$
\begin{aligned}
E_{4}^{(1)}(q) & =\frac{1}{27} a(q) c(q)^{3} \\
E_{4}^{(2)}(q) & =-\frac{1}{3} a(a) b(q)^{3} .
\end{aligned}
$$

Expressing $E_{4}^{(1)}$ and $E_{4}^{(2)}$ in terms of $Q(q)$ and $Q\left(q^{3}\right)$ using (3.6), (3.7) and (3.10), and expressing $a(q), b(q), c(q)$ in terms of $X$ and $Z$ using Definition 11.1 and Lemma 11.2, we get

$$
\begin{aligned}
Q(q)-Q\left(q^{3}\right) & =\frac{80}{9} Z^{4} X, \\
81 Q\left(q^{3}\right)-Q(q) & =80 Z^{4}(1-X) .
\end{aligned}
$$

Solving for $Q(q)$ and $Q\left(q^{3}\right)$ we obtain (11.17) and (11.18).

Next, using Jacobi's discriminant

$$
Q(q)^{3}-R(q)^{2}=1728 q(q ; q)_{\infty}^{24}
$$

(see (18, p. 144) for a simple proof), and making use of (11.11) and (11.17), we obtain

$$
\begin{aligned}
R(q)^{2} & =Q(q)^{3}-1728 q(q ; q)_{\infty}^{24} \\
& =Z^{12}(1+8 X)^{3}-\frac{1728}{27} Z^{12} X(1-X)^{3} \\
& =Z^{12}\left(1-20 X-8 X^{2}\right)^{2} .
\end{aligned}
$$

Taking square roots and comparing the coefficients of $q^{0}$ to determine the sign, we obtain (11.19). Equation (11.20) is obtained in the same way, using (11.12) and (11.18).

\section{Differential equations}

Lemma 12.1. Let $\wp(\theta)=\wp(\theta ; 2 \pi, 2 \pi i t)$ and $q=e^{-2 \pi t}$. Then

$$
\wp^{\prime}(\theta)^{2}=4 \wp(\theta)-\tilde{g}_{2} \wp(\theta)-\tilde{g}_{3},
$$

where ${ }^{2}$

$$
\begin{aligned}
& \tilde{g}_{2}=60 \sum_{(m, n) \neq(0,0)} \frac{1}{(2 \pi n+2 \pi i m t)^{4}}=\frac{Q(q)}{12}, \\
& \tilde{g}_{3}=140 \sum_{(m, n) \neq(0,0)} \frac{1}{(2 \pi n+2 \pi i m t)^{6}}=\frac{R(q)}{216} .
\end{aligned}
$$

\footnotetext{
${ }^{2}$ The Weierstrassian parameters $\tilde{g}_{2}$ and $\tilde{g}_{3}$ should not be confused with our functions $g_{1}$ and $g_{2}$.
} 


\section{Proof}

This is a statement of the differential equation satisfied by the Weierstrass $\wp$ function. See, for example, (8, Ch. 3 and 6$)$ or (19).

Theorem 12.2. Writing $g_{1}=g_{1}(\theta ; q)$ and $g_{2}=g_{2}(\theta ; q)$, we have

$$
\begin{aligned}
& \left(\frac{d g_{1}}{d \theta}\right)^{2}=-\frac{2}{27}\left(g_{1}-\frac{Z}{6}\right)\left(54 g_{1}^{3}+27 Z g_{1}^{2}-Z^{3}(1-X)\right), \\
& \left(\frac{d g_{2}}{d \theta}\right)^{2}=-\frac{2}{9}\left(g_{2}-\frac{Z}{6}\right)\left(54 g_{2}^{3}+27 Z g_{2}^{2}-Z^{3} X\right) .
\end{aligned}
$$

\section{Proof}

Using Lemma 13.5 and the table after Theorem 13.11, observe that Theorem 7.3 may be written in the form

$$
g_{1}-\frac{Z}{6}=\frac{-Z^{3} X}{54\left(\wp+Z^{2} / 12\right)} .
$$

Differentiating and squaring we get

$$
\left(g_{1}^{\prime}\right)^{2}=\frac{Z^{6} X^{2}}{54^{2}} \frac{\left(\wp^{\prime}\right)^{2}}{\left(\wp+Z^{2} / 12\right)^{4}} .
$$

Using Lemma 12.1 and (12.3), this becomes

$$
\left(g_{1}^{\prime}\right)^{2}=\frac{54^{2}}{Z^{6} X^{2}}\left(g_{1}-\frac{Z}{6}\right)^{4}\left(4 \wp^{3}-\frac{Q\left(q^{3}\right)}{12} \wp-\frac{R\left(q^{3}\right)}{216}\right) .
$$

Using (11.18) and (11.20) and rearranging, we get

$$
\begin{gathered}
\left(g_{1}^{\prime}\right)^{2}=\frac{54^{2}}{Z^{6} X^{2}}\left(g_{1}-\frac{Z}{6}\right)^{4}\left(4 \wp^{3}-\frac{Z^{4}(9-8 X)}{108} \wp-\frac{Z^{6}\left(1-\frac{4}{3} X+\frac{8}{27} X^{2}\right)}{216}\right) \\
=\frac{54^{2}}{Z^{6} X^{2}}\left(g_{1}-\frac{Z}{6}\right)^{4}\left(4\left(\wp+\frac{Z^{2}}{12}\right)^{3}-Z^{2}\left(\wp+\frac{Z^{2}}{12}\right)^{2}\right. \\
\left.+\frac{2 X Z^{4}}{27}\left(\wp+\frac{Z^{2}}{12}\right)-\frac{X^{2} Z^{6}}{27^{2}}\right) .
\end{gathered}
$$

Using (12.3) again, this simplifies to

$$
\left(g_{1}^{\prime}\right)^{2}=-4\left(g_{1}-\frac{Z}{6}\right)\left(g_{1}^{3}+\frac{Z}{2} g_{1}^{2}+\frac{Z^{3}(X-1)}{54}\right) .
$$

Rearranging, we complete the proof of the first part of the Theorem.

The second part follows from the first by the modular transformation, using Theorem 4.3 and Lemma 11.2.

Remark 12.4. The first part of Theorem 12.2 was first proved by Berndt et. al. (4, p. 4209, eq. (8.32)). The proof we have given here has also been found independently by Chan and Liu (7). It would be useful to have proof of Theorem 12.2 in the style of Venkatachaliengar (19, pp. 11-13). 


\section{Recurrences for the Eisenstein series $S_{2 n}(q)$ and $E_{2 n}\left(\chi_{3} ; q\right)$}

Expanding the results in Corollary 10.12 in powers of $\theta$ using the series expansions in Theorem 3.1 , we obtain the following results of Liu (15, Theorems 6 and 8):

Theorem 13.1.

$$
\frac{1}{3} E_{2}(q)-E_{2}\left(q^{3}\right)=\frac{1}{4} E_{2}^{(1)}(q)+\frac{1}{12} E_{2}^{(2)}(q)=S_{0}(q)^{2}=E_{0}\left(\chi_{3} ; q\right)^{2},
$$

and, for $n \geq 1$,

$$
\begin{gathered}
\frac{1}{2} E_{2 n+2}^{(1)}(q)=3 S_{0}(q) S_{2 n}(q)+\sum_{k=1}^{n-1}\left(\begin{array}{c}
2 n \\
2 k
\end{array}\right) S_{2 k}(q) S_{2 n-2 k}(q) \\
\frac{1}{6} E_{2 n+2}^{(2)}(q)=3 E_{0}\left(\chi_{3} ; q\right) E_{2 n}\left(\chi_{3} ; q\right)+\sum_{k=1}^{n-1}\left(\begin{array}{l}
2 n \\
2 k
\end{array}\right) E_{2 k}\left(\chi_{3} ; q\right) E_{2 n-2 k}\left(\chi_{3} ; q\right) .
\end{gathered}
$$

Remark 13.4. Equation (13.3) can be deduced from (13.2) (and vice versa) by the modular transformation, using Corollaries 4.7 and 4.9 .

Lemma 13.5. Let $q=e^{-2 \pi t}$. Then

$$
\begin{gathered}
\phi^{\prime}\left(2 \pi i t ; q^{3}\right)=\frac{1}{2}\left(E_{2}(q)-E_{2}\left(q^{3}\right)\right), \\
\wp(2 \pi i t ; 2 \pi, 6 \pi i t)=-\frac{Z^{2}}{12}, \\
\phi^{\prime}\left(\theta ; q^{3}\right)-\phi^{\prime}\left(2 \pi i t ; q^{3}\right)=-\frac{1}{2 \theta^{2}}-\frac{3}{2} S_{0}(q)^{2}+\sum_{n=1}^{\infty} \frac{(-1)^{n}}{(2 n) !} E_{2 n+2}\left(q^{3}\right) \theta^{2 n} .
\end{gathered}
$$

\section{Proof}

From (1.1),

$$
\phi^{\prime}(\theta ; q)=-\frac{1}{8} \csc ^{2} \frac{\theta}{2}+\sum_{n=1}^{\infty} \frac{n q^{n}}{1-q^{n}} \cos n \theta .
$$

Therefore

$$
\begin{aligned}
\phi^{\prime}\left(2 \pi i t ; q^{3}\right) & =\frac{q}{2(1-q)^{2}}+\frac{1}{2} \sum_{n=1}^{\infty} \frac{n q^{3 n}\left(q^{n}+q^{-n}\right)}{1-q^{3 n}} \\
& =\frac{q}{2(1-q)^{2}}-\frac{1}{2} \sum_{n=1}^{\infty} n q^{n}+\frac{1}{2} \sum_{n=1}^{\infty} \frac{n\left(q^{n}+q^{2 n}\right)}{1-q^{3 n}} \\
& =\frac{1}{2} \sum_{n=1}^{\infty} \frac{n\left(q^{n}+q^{2 n}+q^{3 n}\right)}{1-q^{3 n}}-\frac{1}{2} \sum_{n=1}^{\infty} \frac{n q^{3 n}}{1-q^{3 n}} \\
& =\frac{1}{2} \sum_{n=1}^{\infty} \frac{n q^{n}}{1-q^{n}}-\frac{1}{2} \sum_{n=1}^{\infty} \frac{n q^{3 n}}{1-q^{3 n}} \\
& =\frac{1}{2}\left(E_{2}(q)-E_{2}\left(q^{3}\right)\right) .
\end{aligned}
$$

This proves the first part.

The second part follows from the first part, using (7.2) and (11.14). 
Next, using Theorem 3.1 and the first part of the lemma, we get

$$
\begin{aligned}
& \phi^{\prime}\left(\theta ; q^{3}\right)-\phi\left(2 \pi i t ; q^{3}\right) \\
& =-\frac{1}{2 \theta^{2}}+\sum_{n=1}^{\infty} \frac{(-1)^{n-1}}{(2 n-2) !} E_{2 n}\left(q^{3}\right) \theta^{2 n-2}-\frac{1}{2}\left(E_{2}(q)-E_{2}\left(q^{3}\right)\right) \\
& =-\frac{1}{2 \theta^{2}}+\left(\frac{3}{2} E_{2}\left(q^{3}\right)-\frac{1}{2} E_{2}(q)\right)+\sum_{n=1}^{\infty} \frac{(-1)^{n}}{(2 n) !} E_{2 n+2}\left(q^{3}\right) \theta^{2 n} .
\end{aligned}
$$

Applying Theorem 13.1 we complete the proof.

Theorem 13.6. For $n=1,2,3, \cdots$,

$$
\begin{gathered}
S_{2 n+2}(q)=3(2 n+1)(2 n+2) S_{0}(q)^{2} S_{2 n}(q) \\
-2(2 n+1)(2 n+2) \sum_{j=1}^{n-1}\left(\begin{array}{c}
2 n \\
2 j
\end{array}\right) S_{2 j}(q) E_{2 n+2-2 j}\left(q^{3}\right), \\
E_{2 n+2}\left(\chi_{3} ; q\right)=-9(2 n+1)(2 n+2) E_{0}\left(\chi_{3} ; q\right)^{2} E_{2 n}\left(\chi_{3} ; q\right) \\
-2(2 n+1)(2 n+2) \sum_{j=1}^{n-1}\left(\begin{array}{c}
2 n \\
2 j
\end{array}\right) E_{2 j}\left(\chi_{3} ; q\right) E_{2 n+2-2 j}(q) .
\end{gathered}
$$

\section{Proof}

Expand both sides of Theorem (7.1) in powers of $\theta$ using Theorem 3.1 and Lemma 13.5, and equate coefficients of $\theta^{2 n}$. This proves the first part. The second part follows from the first, using the transformation $t \rightarrow 1 / 3 t$.

Remark 13.7. Chan and Liu (7) have obtained a formula for $S_{2 n}$ purely in terms of $S_{2 k}$, with $k<n$, by differentiating the first result in Theorem 12.2.

Lemma 13.8. For $n=2,3,4, \cdots$,

$$
\begin{aligned}
E_{2 n}(q) & =\sum_{2 j+3 k=n} K_{j, k} Q(q)^{j} R(q)^{k}, \\
E_{2 n}\left(q^{3}\right) & =Z^{2 n} p_{n}(X),
\end{aligned}
$$

where $K_{j, k}$ are rational numbers, and $p_{n}(X)$ is a polynomial in $X$ with rational coefficients and degree $\lfloor 2 n / 3\rfloor$.

\section{Proof}

A proof of (13.9) has been given by Ramanujan (18, p. 141). Equation (13.10) follows from (13.9) by induction and making use of (11.18) and (11.20).

Theorem 13.11. $S_{0}(q)=E_{0}\left(\chi_{3} ; q\right)=\frac{Z}{6}$, and for $n=1,2,3, \cdots$,

$$
\begin{aligned}
S_{2 n}(q) & =Z^{2 n+1} P_{n}(X), \\
E_{2 n}\left(\chi_{3} ; q\right) & =3^{n} Z^{2 n+1} P_{n}(1-X),
\end{aligned}
$$

where $P_{n}$ is a polynomial with rational coefficients and degree $\leq\lfloor(2 n+1) / 3\rfloor$. 


\section{Proof}

Equation (13.12) follows by induction from Theorem 13.6, using Lemma 13.8. The bound on the degree of $P_{n}$ follows because

$$
\left\lfloor\frac{2 j}{3}\right\rfloor+\left\lfloor\frac{2 n+1-2 j}{3}\right\rfloor \leq\left\lfloor\frac{2 n+1}{3}\right\rfloor .
$$

Equation (13.13) follows from (13.12) using Corollary 4.7 and Lemma 11.2.

The first few instances of Theorem 13.11 are as follows:

$$
\begin{aligned}
& S_{0}=\frac{1}{6} Z \\
& S_{2}=\frac{1}{27} Z^{3} X \\
& S_{4}=\frac{1}{27} Z^{5} X \\
& S_{6}=\frac{1}{27} Z^{7} X\left(1+\frac{4}{3} X\right) \\
& S_{8}=\frac{1}{27} Z^{9} X\left(1+8 X+\frac{80}{81} X^{2}\right) \\
& S_{10}=\frac{1}{27} Z^{11} X\left(1+36 X+\frac{848}{27} X^{2}\right) \\
& S_{12}=\frac{1}{27} Z^{13} X\left(1+\frac{448}{3} X+\frac{12448}{27} X^{2}+\frac{6080}{81} X^{3}\right) \\
& S_{14}=\frac{1}{27} Z^{15} X\left(1+604 X+\frac{422432}{81} X^{2}+\frac{289792}{81} X^{3}+\frac{70400}{729} X^{4}\right) \\
& E_{0}\left(\chi_{3}, q\right)=\frac{1}{6} Z \\
& E_{2}\left(\chi_{3}, q\right)=\frac{1}{9} Z^{3}(1-X) \\
& E_{4}\left(\chi_{3}, q\right)=\frac{1}{3} Z^{5}(1-X) \\
& E_{6}\left(\chi_{3}, q\right)=Z^{7}(1-X)\left(1+\frac{4}{3}(1-X)\right) \\
& E_{8}\left(\chi_{3}, q\right)=3 Z^{9}(1-X)\left(1+8(1-X)+\frac{80}{81}(1-X)^{2}\right) \\
& E_{10}\left(\chi_{3}, q\right)=9 Z^{11}(1-X)\left(1+36(1-X)+\frac{848}{27}(1-X)^{2}\right) \\
& \left.E_{12}, q\right)=27 Z^{13}(1-X)\left(1+\frac{448}{3}(1-X)+\frac{12448}{27}(1-X)^{2}\right. \\
& =
\end{aligned}
$$




\section{References}

[1] G. E. Andrews, R. Askey and R. Roy, Special functions, Encyclopedia of Mathematics and its Applications, 71. Cambridge University Press, Cambridge, 1999.

[2] B. C. Berndt, Ramanujan's Notebooks, Part III, Springer-Verlag, 1991.

[3] B. C. Berndt, Ramanujan's Notebooks, Part V, Springer-Verlag, 1998.

[4] B. C. Berndt, S. Bhargava and F. G. Garvan, Ramanujan's theories of elliptic functions to alternative bases, Trans. Amer. Math. Soc., 347 (1995), 4163-4244. Reprinted in (3, Chapter 33, pp. 89-181)

[5] J. M. Borwein and P. B. Borwein, A cubic counterpart of Jacobi's identity and the AGM, Trans. Amer. Math. Soc. 323 (1991), no. 2, 691-701.

[6] J. M. Borwein, P. B. Borwein and F. G. Garvan, Some cubic modular identities of Ramanujan, Trans. Amer. Math. Soc. 343 (1994), no. 1, 35-47.

[7] H. H. Chan and Z.-G. Liu, Analogues of Jacobi's inversion formula for the incomplete elliptic integral of the first kind, Advances in Mathematics 174 (2003), 69-88.

[8] K. Chandrasekharan, Elliptic functions, Springer-Verlag, Berlin, 1985.

[9] S. Cooper, The development of elliptic functions according to Ramanujan and Venkatachaliengar, Proceedings of The International Conference on the Works of Srinivasa Ramanujan (C. Adiga and D. D. Somashekara, eds.), University of Mysore, Manasagangotri, Mysore - 570 006, India (2001) 81-99.

Available electronically at http://iims.massey.ac.nz/research/letters/ (see vol. 1, 2000)

[10] S. Cooper, On sums of an even number of squares, and an even number of triangular numbers: an elementary approach based on Ramanujan's ${ }_{1} \psi_{1}$ summation formula, $q$-Series with Applications to Combinatorics, Number Theory and Physics (B. C. Berndt and K. Ono, eds.), Contemporary Mathematics, No. 291, American Mathematical Society, Providence, RI (2001) $115-137$.

[11] S. Cooper, Cubic theta functions, Journal of Computational and Applied Mathematics, to appear.

[12] M. Hirschhorn, F. Garvan and J. Borwein, Cubic analogues of the Jacobian theta functions $\theta(z, q)$, Canadian J. Math. 45 (4), 1993, 673-694.

[13] A. W. Knapp, Elliptic curves, Mathematical Notes, 40. Princeton University Press, Princeton, NJ, 1992

[14] M. I. Knopp, Modular functions in analytic number theory, Markham Publishing Co., Chicago, Ill. 1970.

[15] Zhi-Guo Liu, Some Eisenstein series identities associated with the Borwein functions, Symbolic computation, number theory, special functions, physics and combinatorics (Gainesville, FL, 1999), 147-169, Dev. Math., 4, Kluwer Acad. Publ., Dordrecht, 2001.

[16] S. Ramanujan, On certain arithmetical functions, Trans. Camb. Phil. Soc., 22 (9), 1916, 159-184. Reprinted in (18, 136-162).

[17] S. Ramanujan, Notebooks, (2 volumes), Tata Institute of Fundamental Research, Bombay, 1957. 
[18] S. Ramanujan, Collected papers, AMS Chelsea Publishing, Providence, Rhode Island, 2000.

[19] K. Venkatachaliengar, Development of Elliptic Functions according to Ramanujan, Department of Mathematics, Madurai Kamaraj University, Technical Report 2, 1988.

[20] E. T. Whittaker and G. N. Watson, A course of modern analysis, Reprint of the fourth (1927) edition. Cambridge University Press, Cambridge, 1996. 